\title{
The TNF-family cytokine TL1A drives IL-13-dependent small intestinal inflammation
}

\author{
F Meylan ${ }^{1,6}$, Y-J Song ${ }^{1,6}$, I Fuss $^{2}$, S Villarreal ${ }^{1}$, E Kahle $^{1}$, I-J Malm ${ }^{1}$, K Acharya $^{1}$, HL Ramos ${ }^{3}$, L Lo $^{4}$, \\ MM Mentink-Kane ${ }^{5}$, TA Wynn ${ }^{5}$, T-S Migone ${ }^{4}$, W Strober ${ }^{2}$ and RM Siegel ${ }^{1}$
}

The tumor necrosis factor (TNF)-family cytokine TL1A (TNFSF15) costimulates T cells through its receptor DR3 (TNFRSF25) and is required for autoimmune pathology driven by diverse T-cell subsets. TL1A has been linked to human inflammatory bowel disease (IBD), but its pathogenic role is not known. We generated transgenic mice that constitutively express TL1A in T cells or dendritic cells. These mice spontaneously develop IL-13-dependent inflammatory small bowel pathology that strikingly resembles the intestinal response to nematode infections. These changes were dependent on the presence of a polyclonal T-cell receptor (TCR) repertoire, suggesting that they are driven by components in the intestinal flora. Forkhead box P3 (FoxP3)-positive regulatory T cells (Tregs) were present in increased numbers despite the fact that TL1A suppresses the generation of inducible Tregs. Finally, blocking TL1A-DR3 interactions abrogates 2,4,6 trinitrobenzenesulfonic acid (TNBS) colitis, indicating that these interactions influence other causes of intestinal inflammation as well. These results establish a novel link between TL1A and interleukin 13 (IL-13) responses that results in small intestinal inflammation, and also establish that TL1A-DR3 interactions are necessary and sufficient for T cell-dependent IBD.

\section{INTRODUCTION}

Interactions between tumor necrosis factor (TNF)-family receptors and their ligands have major roles in shaping critical features of immune responses, including programmed cell death and lymphocyte costimulation. DR3 (TNFRSF25/ TRAMP/LARD/WSL-1) is one such TNF-family receptor that like TNF-R1 contains a death domain and can activate nuclear factor- $\kappa \mathrm{B}$ and mitogen-activated protein kinases, or caspases and apoptosis, depending on the cellular context. ${ }^{1,2}$ However, unlike TNF-R1, T cells express the highest levels of DR3. TL1A, the TNF-family ligand for DR3, costimulates T-cell proliferation and cytokine production in vitro. ${ }^{3}$ TL1A expression is highly regulated and requires induction by TNF- $\alpha$ and interleukin$1 \beta$ (IL-1 $\beta$ ) in endothelial cells, ${ }^{3}$ and Toll-like receptor or FcR stimulation in myeloid cells. ${ }^{4-6}$ T-cell receptor (TCR) stimulation can also induce expression of TL1A in T cells with slower kinetics. ${ }^{4}$ Such selective expression of TL1A may explain the fact that DR3-deficient mice are resistant to multiple mouse models of $\mathrm{T}$ cell-mediated autoimmune disease, but generally display normal systemic responses to the antigens used to induce these autoimmune diseases. ${ }^{4-6}$

Recently, several reports have suggested a role for TL1A and DR3 in inflammatory bowel disease (IBD). ${ }^{7,8}$ Increased expression of both TL1A and DR3 was reported in the lamina propria in biopsies of patients with ulcerative colitis and Crohn's disease, with the level of TL1A expression correlating with the severity of the inflammation. In addition, elevated TL1 A and DR3 expression was found in two different animal models of IBD: SAMP1/YitFc and TNF $\triangle$ ARE ${ }^{8}$ Single-nucleotide polymorphisms in the TL1A locus have also been linked to Crohn's disease in multiple genome-wide scans. ${ }^{9-11}$ Although these data provide strong circumstantial evidence that TL1A is involved in the pathogenesis of IBD, the causal role of TL1A-DR3 interactions in IBD is not known. To address this issue, we have generated several lines of TL1A transgenic mice that constitutively express TL1A in T cells and dendritic cells (DCs) at levels similar to that observed when cells are induced to express TL1A by proinflammatory stimuli, and studied the effects of

\footnotetext{
${ }^{1}$ Immunoregulation Section, Autoimmunity Branch, NIAMS, Bethesda, Maryland, USA. ${ }^{2}$ Mucosal Immunology Section, Laboratory of Host Defenses, NIAID, Bethesda, Maryland, USA. ${ }^{3}$ Molecular Immunology and Inflammation Branch, NIAMS, Bethesda, Maryland, USA. ${ }^{4}$ Human Genome Sciences, Rockville, MD, USA. ${ }^{5}$ Immunopathogenesis Section, Laboratory of Parasitic Diseases, NIAID, NIH Bethesda, Bethesda, Maryland, USA. ${ }^{6}$ These authors contributed equally to this work. Correspondence: RM Siegel (siegelr@mail.nih.gov)

Received 5 March 2010; accepted 1 September 2010; published online 27 October 2010. doi:10.1038/mi.2010.67
} 
a

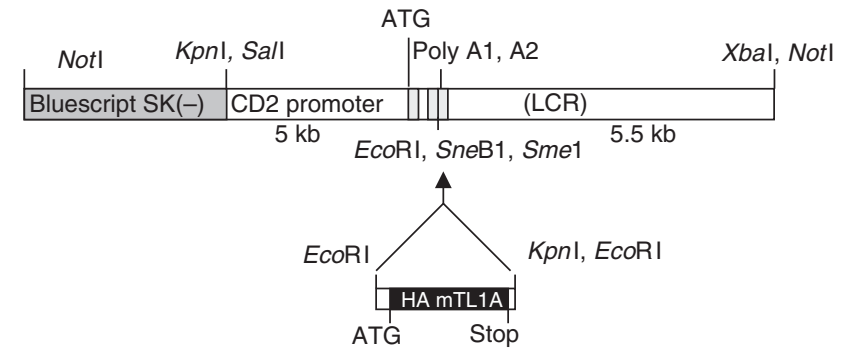

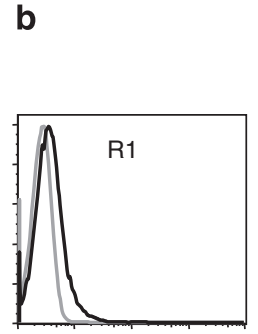
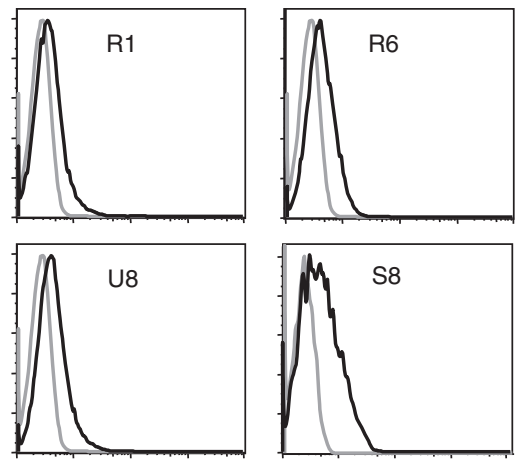

C
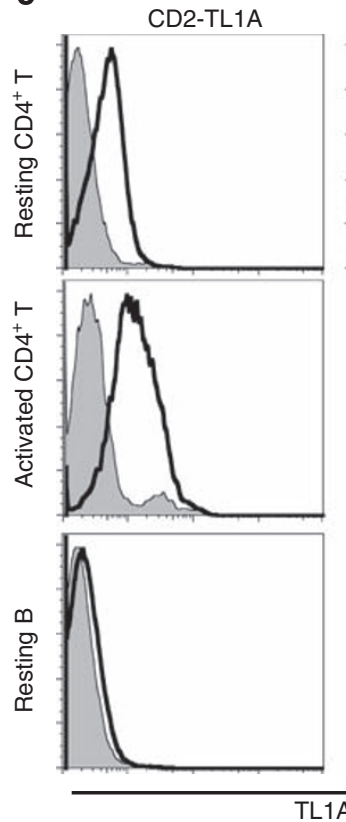
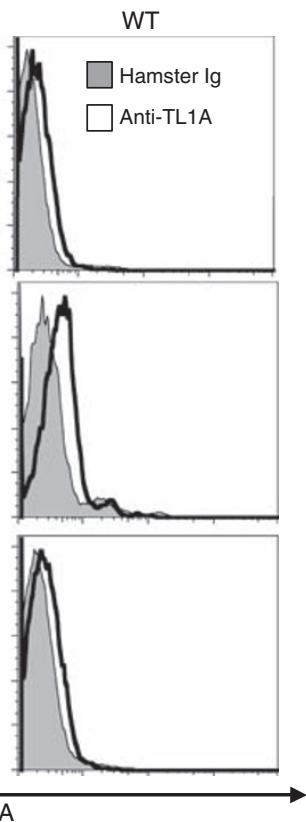

Figure 1 Generation of and TL1A expression in CD2-TL1A transgenic mice. (a) Schematic of CD2-TL1A transgenic construct illustrating placement of the mouse TL1A-hemagglutinin (HA) complementary DNA (cDNA) in the CD2 promoter/enhancer cassette. (b) Comparison of transgene expression in four independent founder lines of CD2-TL1A transgenic mice assayed by intracellular flow cytometry of anti-HA on CD3-gated splenocytes from transgenic (thick) and control (thin) mice. (c) Transgenic vs. endogenous cell surface TL1A expression assayed by flow cytometry with anti-TL1A monoclonal antibody (mAb) $5.4 \mathrm{G} 6$ vs. control hamster Ig on resting CD4+ $\mathrm{T}$ cells isolated from spleen and lymph nodes (top), on CD4 ${ }^{+} \mathrm{T}$ cells $48 \mathrm{~h}$ after activation with anti-CD3/anti-CD28 (middle), and on resting B cells gated on B220 (bottom) from line R6 CD2-TL1A transgenic and control $\mathrm{C} 57 \mathrm{BL} / 6$ mice. The color reproduction of this figure is available on the html full text version of the manuscript.

TL1A blockade during induction of a hapten-driven model of inflammatory colitis.

\section{RESULTS}

In transgenic mice with a hemagglutinin epitope-tagged murine TL1A complementary DNA linked to the CD2 promoter/ enhancer cassette, ${ }^{12}$ four founder lines had detectable levels of TL1A expression in T cells assayed by flow cytometry (Figure 1a and $\mathbf{b}$ ). Line R6 was selected for in-depth analysis. To confirm expression of surface TL1A on transgenic T cells and compare with endogenous levels, we generated monoclonal anti-mouse TL1A antibodies (Supplementary Figure S1A online). Staining of resting and activated $\mathrm{T}$ cells with monoclonal antibody $(\mathrm{mAb})$ 5G4.6 revealed levels of surface TL1A on resting CD2-TL1A transgenic $\mathrm{T}$ cells that are higher than that present on wild-type T cells after activation with anti-CD3 and anti-CD28 (Figure 1c). Activation of transgenic $\mathrm{T}$ cells resulted in increased transgenic and endogenous surface TL1A (Figure 1c). B cells expressed only very low levels of endogenous TL1A that was not increased in transgenic cells (Figure 1c, bottom panels). Thus, the mice generated using the CD2-mTL1A cassette constitutively expressed TL1A at levels at least as high as peak levels on activated T cells.

\section{T-cell activation and accelerated memory T-cell generation in TL1A transgenic mice}

To investigate whether constitutively expressed TL1A can costimulate $\mathrm{T}$ cells in vivo, we examined the lymphoid compartment and the state of T-cell activation in CD2-TL1A transgenic mice. We found mild splenomegaly and mild-to-moderate mesenteric lymphadenopathy in adult mice of each of the CD2TL1A transgenic lines (Figure 2a). Flow cytometric analysis revealed increased numbers of $\mathrm{CD} 4^{+} \mathrm{T}$ cells in mesenteric lymph nodes (mLNs) and to a lesser extent in spleen (Figure $\mathbf{2 b}$ ). $\mathrm{CD}^{+} \mathrm{T}$-cell yields were mildly increased in mLNs and normal in the spleen. The B-cell and myeloid cell numbers were not affected (data not shown). Percentages of cells expressing CD69, a marker of recent activation, and also memory T-cell markers 

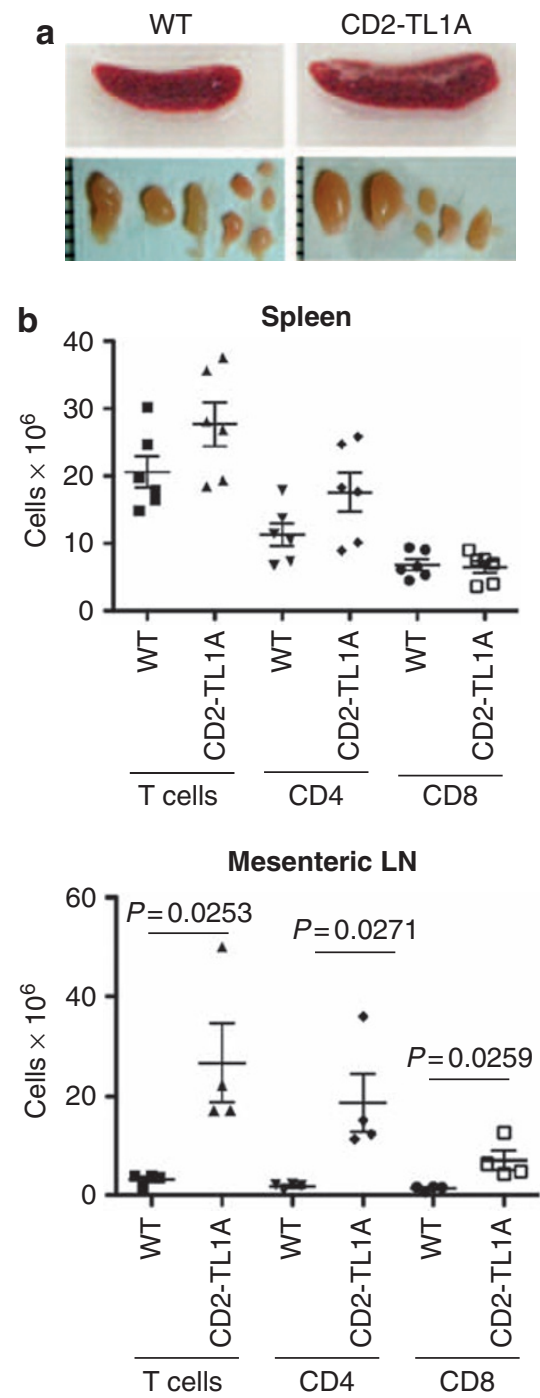
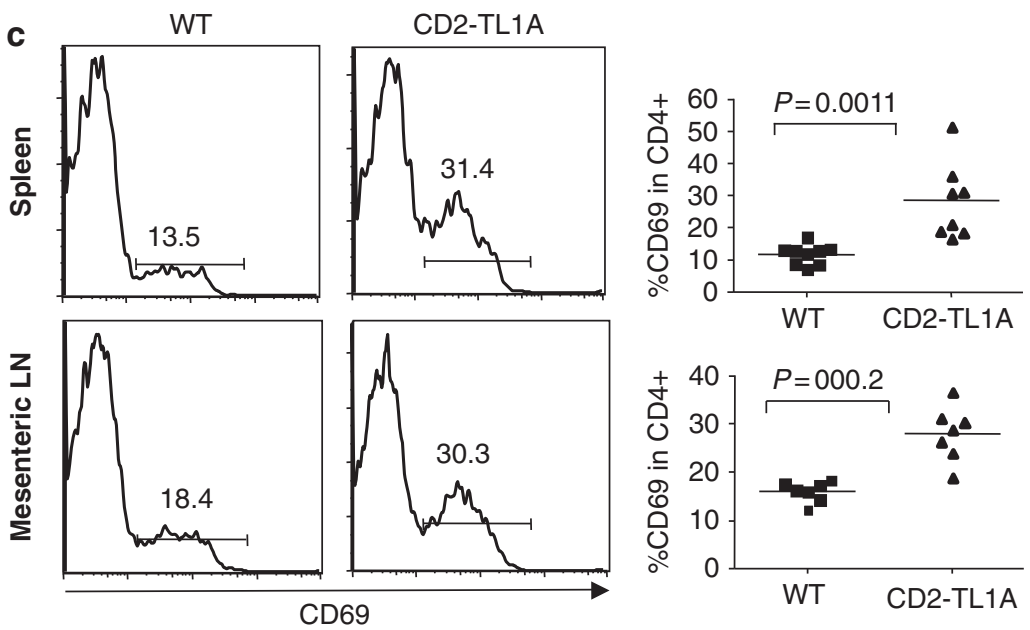

d
के
के

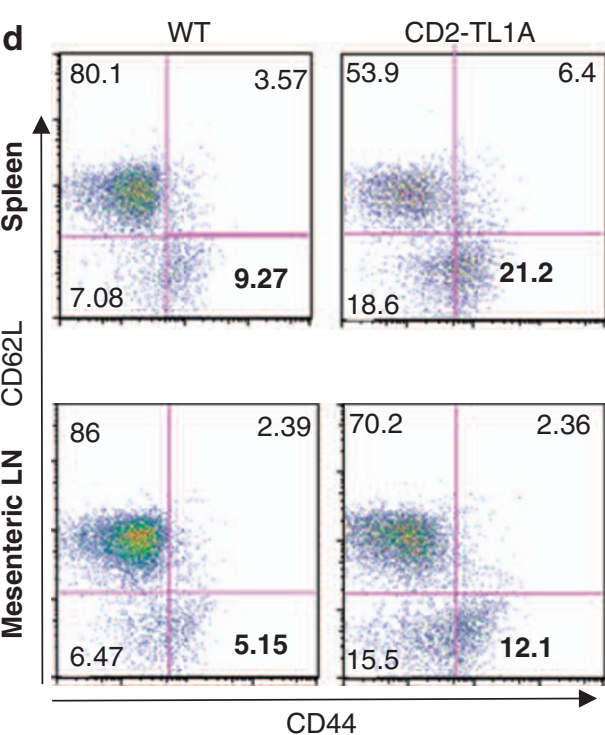

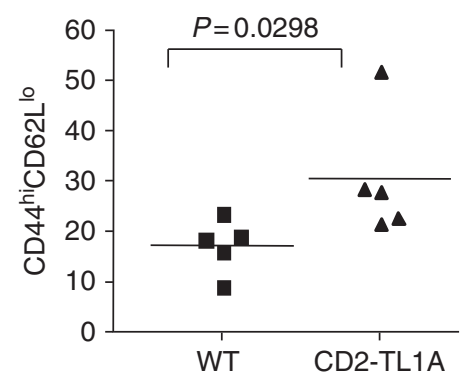

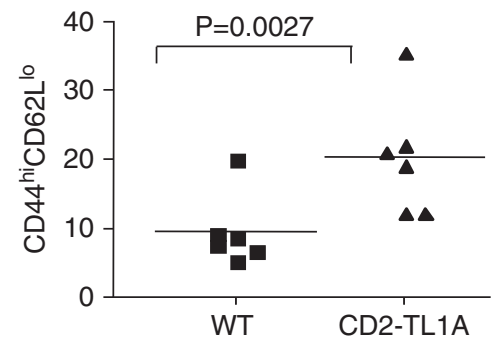

Figure 2 Characterization of the T-cell compartment in TL1A transgenic mice. (a) Gross appearance of spleen and mesenteric lymph nodes (mLNs) from C57BL/6 wild-type and line R6 CD2-TL1A transgenic mice $>3$ months of age. (b) Absolute number of T cells, CD4 ${ }^{+} \mathrm{T}$ cells, and CD8 ${ }^{+} \mathrm{T}$ cells from spleen and $\mathrm{mLNs}$ from line R6 CD2-TL1A transgenic mice 20-24 weeks old and age-matched controls. (c) Representative flow cytometric profiles and compilation of the percentage of $\mathrm{CD} 69^{+}$cells as a percentage of CD4 ${ }^{+} \mathrm{T}$ cells in spleen and mLNs from line R6 CD2-TL1A transgenic mice 10-16 weeks of age and age-matched controls. (d) Representative flow cytometric profiles and compilation of the percentage of memory $\mathrm{CD} 44^{\text {hi }} \mathrm{CD} 62 \mathrm{~L}^{\text {lo }}$ cells as a percentage of CD4 ${ }^{+} \mathrm{T}$ cells in spleen and $\mathrm{mLNs}$ from line R6 CD2-TL1A transgenic mice 10-16 weeks old and age-matched controls. Statistical analysis for comparison of percentages of the indicated subsets in transgenic and control mice was performed by unpaired two-tailed Student's $t$-test.

were elevated in $\mathrm{CD} 4{ }^{+} \mathrm{T}$ cells in spleen and mLNs (Figure 2c and $\mathbf{d}$ ), and to a lesser extent in $\mathrm{CD} 8^{+} \mathrm{T}$ cells (Supplementary Figure S2 online). Thus, TL1A can costimulate T cells to drive increased activation and memory cell formation in vivo without exogenous stimuli in CD2-TL1A transgenic mice.

\section{Spontaneous intestinal inflammation in CD2-TL1A transgenic mice}

Inspection of the intestines of CD2-TL1A transgenic mice of $>6$ weeks of age revealed $100 \%$ incidence of visible small intestinal inflammatory changes, including bowel wall thickening (Figure 3a), dilatation, and lengthening that progressed with age and culminated in massive ileal dilatation in many animals at $\geqslant 6$ months of age (Figure 3a, bottom panel). Remarkably, the colons of these mice had no macroscopic evidence of inflammation. Histological examination revealed inflammatory changes in the small intestine that, when fully developed, included thickening of the muscularis and submucosa, lengthening, and/or distorted villi accompanied by infiltration of the lamina propria with inflammatory cells, including lymphocytes, macrophages, and neutrophils. Finally, there was striking hyperplasia of goblet cells, particularly in the jejunum and ileum (Figure 3b). Quantitative scoring of inflammatory changes using a previously described scoring system ${ }^{13}$ revealed significant inflammation present in the small intestine, with the most severe changes in the ileum and no significant inflammation in the colon (Figure 3c). Similar severity of histological changes was found in the ileum of most founder lines when the disease was fully developed at 6 months of age 
a

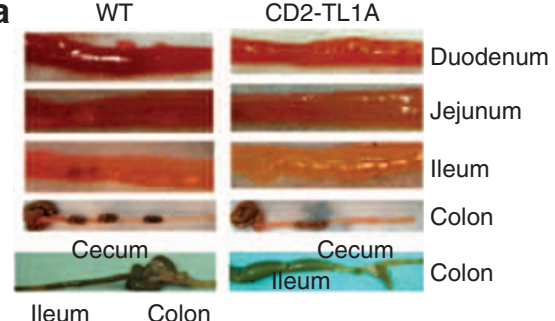

b

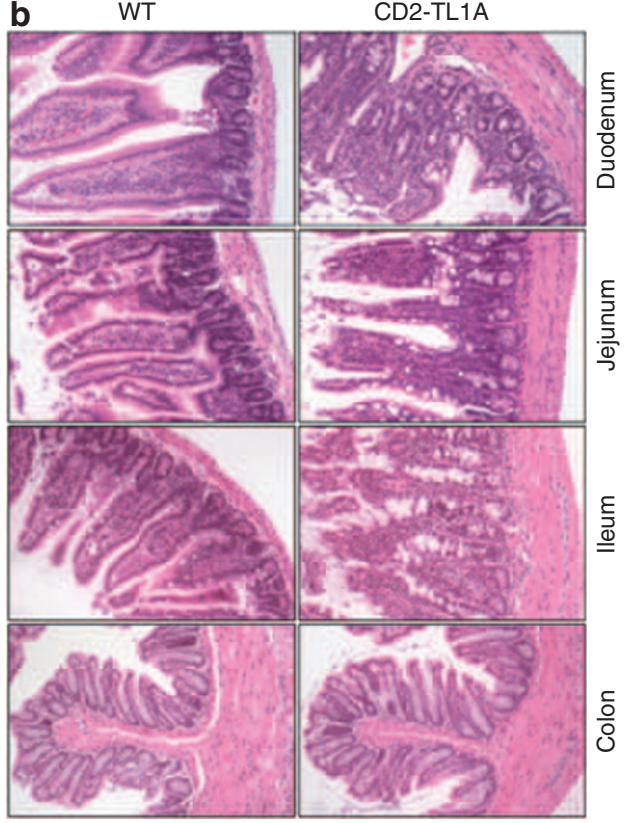

c

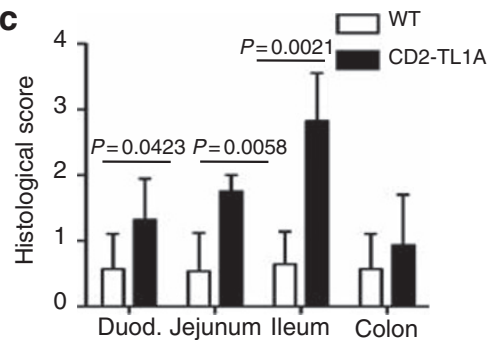

d $\frac{P=0.0096}{\frac{P=0.0097}{P=0.0075}}$

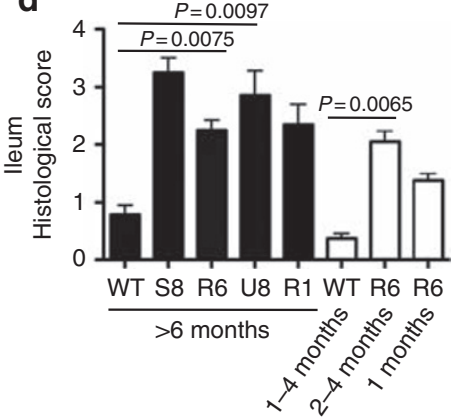

e

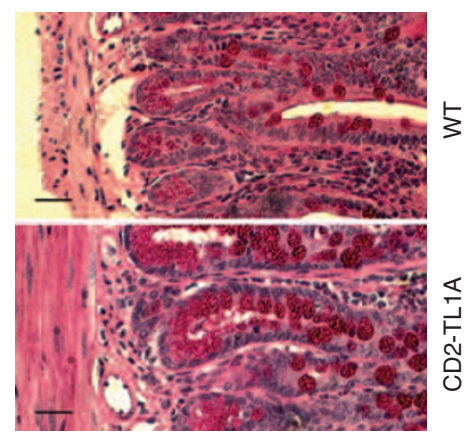

f
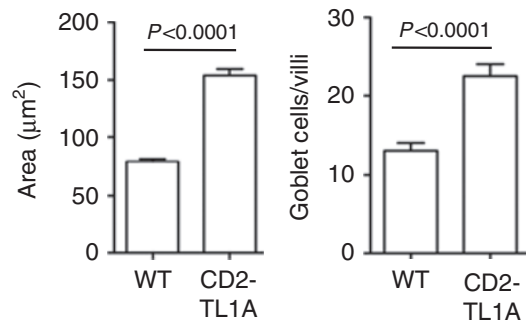

g
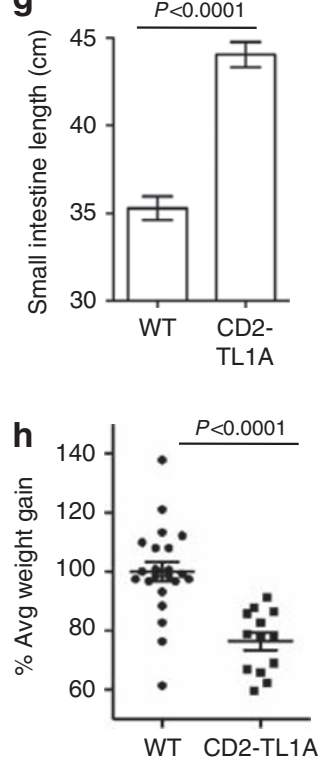

Figure 3 TL1A transgenics develop spontaneous inflammatory bowel disease. (a) Gross appearance of the indicated sections of intestine from 3-month-old line R6 CD2-TL1A transgenic ( $\mathrm{Tg}$ ) mice and age-matched controls, with an example of the appearance of the grossly distended ileum from 1.5-year-old CD2-TL1ATg mice, compared with aged-matched control mice in the bottom panel. (b) Hematoxylin and eosin (H\&E)-stained tissue sections of the indicated portions of intestine from a 3-month-old line R6 CD2-TL1ATg mouse compared with a littermate control. (c) Compilation of histological scores of all four founders $>6$ months of age for each section. Each section was scored by an observer blinded to the genotype of the mouse. Statistical analysis for comparison of transgenic and control mice was performed using Mann-Whitney test. Sections from at least four mice per group were scored. (d) Comparison of histological scores from the ileum of mice from four independent founders of CD2-TL1ATg mice, with at least five sections scored for each group of the indicated age (except for line S8 and 1-month-old line R6, $n=2$ ). Statistical analysis for comparison of transgenic and control mice was performed using Mann-Whitney test. (e) Goblet cell hyperplasia in the ileum of CD2-TL1A line R6 transgenic mouse and wild-type (WT) control. Periodic acid-Schiff (PAS) staining, scale bar $=50 \mu \mathrm{M}$. (f) Quantitation using ImageJ software (NIH, Bethesda, MD) of average goblet cell area and number of goblet cells/villus in sections of ileum from CD2-TL1A line R6 transgenic mice and age-matched controls $(n=5)$. (g) Small intestinal length (measured from the pylorus to ileocecal valve) of adult WT and CD2-TL1ATg mice ( $n>5$ each group). Statistical analysis for comparison between transgenic and control mice was performed using unpaired two-tailed Student's $t$-test. (h) Comparison of weight gain for the 14 days after weaning of CD2-TL1ATg mice (line R6). Statistical analysis for comparison between transgenic and control mice was performed using unpaired two-tailed Student's $t$-test.

(Figure 3d). Within line R6, which was examined in more detail, pathology worsened as mice aged (Figure 3d). Periodic acidSchiff staining of the ileum revealed remarkable increase in the number and size of goblet cells (Figure $3 \mathbf{e}$ and $\mathbf{f}$ ). The total length of the small intestine was significantly increased in CD2-TL1A transgenic mice compared with age-matched controls (Figure 3g), likely as a consequence of goblet cell and smooth muscle hyperplasia. At 2 weeks of age, T-cell activation and memory markers were similar to controls and no small intestinal inflammation could be seen, whereas at 4 weeks of age, goblet cell hyperplasia and increased $\mathrm{CD} 9^{+}$and memory phenotype $\mathrm{T}$ cells could be seen
(Supplementary Figure S3 online). CD2-TL1A transgenic mice also gained weight at a significantly lower rate in the 2 weeks following weaning compared with wild-type littermates (Figure 3h), indicating that the small intestinal pathology had clinical consequences.

\section{Dose-dependent effects of deregulated expression of TL1A in DCs}

In addition to being expressed on activated T cells, TL1A expression can be induced in activated DCs by Toll-like receptor and Fc-receptor signaling. ${ }^{4,14,15}$ To determine whether TL1A 
expressed by DCs can result in pathological consequences similar to T cell-expressed TL1A, we also produced transgenic mouse lines expressing murine TL1A under the control of the $\mathrm{CD} 11 \mathrm{c}$ promoter ${ }^{16} \mathrm{CD} 11 \mathrm{c}$-TL1A transgenic mouse lines exhibited a much wider range of mTL1A expression (3- to 65-fold) compared with endogenous cells than did CD2-TL1A transgenic lines (Supplementary Figure S4A online). TL1A expression in the lines expressing the highest levels of TL1A was similar to peak levels of TL1A expression in mouse DCs stimulated with lipopolysaccharide or immunoglobulin crosslinking. ${ }^{4}$ However, when normalized to expression in resting T cells, TL1A mRNA in DCs of CD11c transgenic mice was at least an order of magnitude lower than that observed on T cells in CD2-TL1A transgenic lines (Supplementary Figure S4B online). In founder lines expressing TL1A on DCs more than eightfold higher than endogenous levels, we also noted inflammatory changes in the small intestine with similar pathological features to that in CD2-TL1A transgenic mice, including goblet cell hyperplasia. Pathological changes were generally milder and more restricted to the ileum than in the CD2-TL1A transgenic mice. Inflammation was accompanied by lengthening of the small intestine and failure to gain weight after weaning, with the severity depending on transgene expression level (Supplementary Figure S4D-F online). Mice expressing less than eightfold more TL1A than wild-type DCs (TL1A Lo) had less severe histological changes and no significant increases in small intestinal length or impairment of weight gain after weaning. This suggests that the absolute level, rather than the source of TL1A, is the most important factor in triggering intestinal inflammation.

\section{Intestinal pathology in CD2-TL1A transgenic mice is dependent on IL-13 and DR3}

To determine which cytokines are associated with the small intestinal inflammation observed in TL1A-transgenic mice, we performed real-time quantitative reverse transcriptase-PCR on RNA isolated from different sections of the intestine of CD2TL1A transgenic mice and wild-type littermates between 8 weeks and 6 months of age (Figure 4a). The most striking abnormality was 20 - to 50-fold elevation of IL-13 mRNA in tissue from all sections of the small intestine. Elevation of IL-13 was accompanied by increases in IL-5, but not IL- 4 mRNA, and therefore did not represent a typical Th2 response (data not shown). IL-17 mRNA levels were elevated to a lesser extent than IL-13, and only in the jejunum and duodenum. Expression of IL-22, a member of the IL-10 family that is known to be coexpressed by some IL-17-secreting T cells, and the IL-23 receptor, which is expressed on Th17 cells, were also not increased above baseline (data not shown). This suggests that either $\mathrm{T}$ cells producing IL-17 but not IL-22 or another cell type may be producing IL-17 in the intestine of CD2-TL1A transgenic mice. Strikingly, interferon- $\gamma($ IFN- $\gamma$ ) expression was not elevated above baseline in any areas of the intestine in TL1A transgenic mice (Figure 4a). IL-13 expression was accompanied by an age-associated accumulation of mast cells in the submucosa and serosal layers of the intestine, as seen in other types of intestinal inflammation associated with Th2 responses (Figure 4b). Levels of IL-13R $\alpha 2$ protein and saturation of IL-13R $\alpha 2$ with IL-13, which are indicators of IL-13 activity, ${ }^{17}$ were both elevated in the serum of TL1A transgenic mice (Figure 4c). Taken together, these data identify IL-13 as a prominent cytokine induced by TL1A in biologically significant amounts.

The increased numbers of T cells in the mLNs suggested that there might be increased trafficking of T cells between the intestinal lamina propria and mLNs. To determine if this was the case, we measured the percentage of T cells expressing the chemokine receptor CCR9 (chemokine (C-C motif) receptor 9), a known marker of $\mathrm{T}$ cells that home to the lamina propria. ${ }^{18}$ CCR9 expression was markedly elevated in the mLNs but not in the spleen of CD2-TL1A transgenic mice (Supplementary Figure S5A online). Confirming this idea, the percentage of $\mathrm{CD} 4{ }^{+} \mathrm{T}$ cells in preparations of lamina propia from CD2-TL1A transgenic mice was markedly increased compared with controls (Supplementary Figure S5B online). Immunofluorescence confirmed the accumulation of $\mathrm{CD}^{+}{ }^{+}$lymphocytes in the lamina propria, particularly in the tips of enlarged villi (Supplementary Figure S5C online). To determine what effector T-cell subsets are promoted by chronic TL1A stimulation, we isolated T cells from spleen, mLNs, and the small intestinal lamina propria, and then measured intracellular cytokine production after stimulation with phorbol myristate acetate and ionomycin. We observed increases in IL-17, IL-4, and IFN- $\gamma$-producing $\mathrm{CD}^{+}{ }^{+} \mathrm{T}$ cells in the mLNs and spleen of TL1A transgenic mice, with increases in IL-17-producing T cells being the most significant (Figure 4d). In $\mathrm{T}$ cells isolated from the lamina propria, the frequency of IL-17, IL-13, and IL-4-producing cells was slightly increased, and IFN- $\gamma$-producing cells was decreased (Supplementary Figure S5D online). However, as $\mathrm{CD} 4{ }^{+} \mathrm{T}$-cell yields were increased in all of these tissues from CD2-TL1A transgenic mice, the absolute number of effector $\mathrm{T}$ cells is likely to be relatively higher than in controls. TL1A overexpression can therefore promote accumulation of many types of effector $\mathrm{T}$ cells in the intestinal lamina propria and mLNs, and to a lesser extent systemically.

To determine whether IL-13 or IL-17 were pathogenic in the small intestinal inflammation driven by TL1A, we treated cohorts of CD2-TL1A transgenic mice with neutralizing antibodies against IL-13 and IL-17, beginning at 2 weeks after birth. Anti-IL-13 was highly effective in reducing inflammatory pathology, whereas neutralizing IL-17 had little effect (Figure 4e), suggesting that IL-13 is a key pathogenic cytokine in the intestinal inflammation induced by chronic TL1A expression.

The TNF receptor DR3 is thought to mediate most, but not all, of the biological effects of TL1A.,6,19 To determine which effects of constitutive TL1A expression that we observed above were because of stimulation through DR3, we crossed CD2TL1A transgenic mice to DR3-deficient mice. ${ }^{20}$ The increased CD69 expression seen in TL1A transgenic mice was ablated on a DR3-deficient background (Figure 5a). The small and large bowels had a normal appearance in TL1ATg $\times$ DR3KO mice, and as shown in Figure 5b, the gross and microscopic features of the inflammatory pathology in the ileum driven by TL1A were absent on the DR3KO background. IL-13 levels were 
a

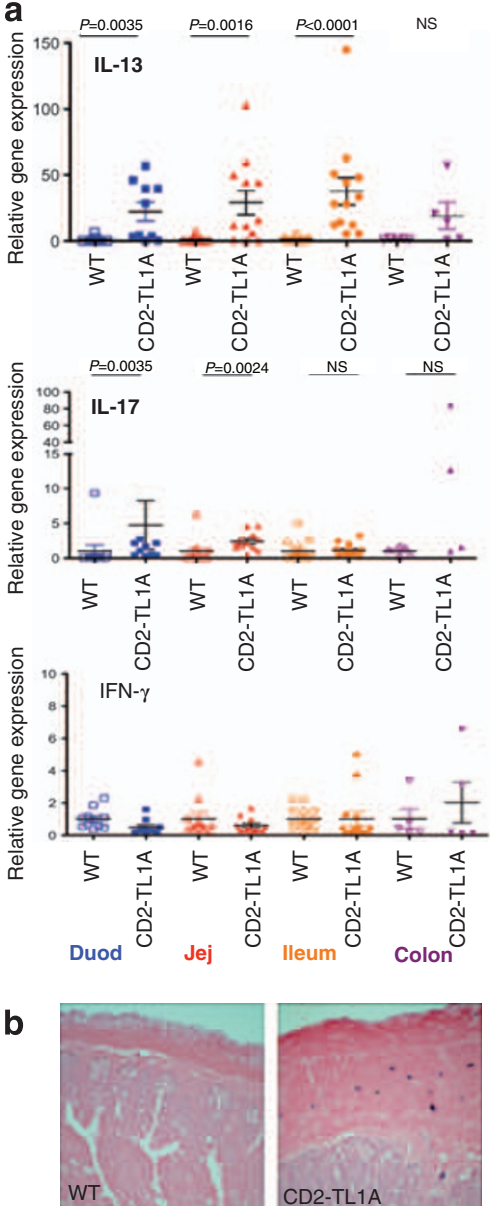

C
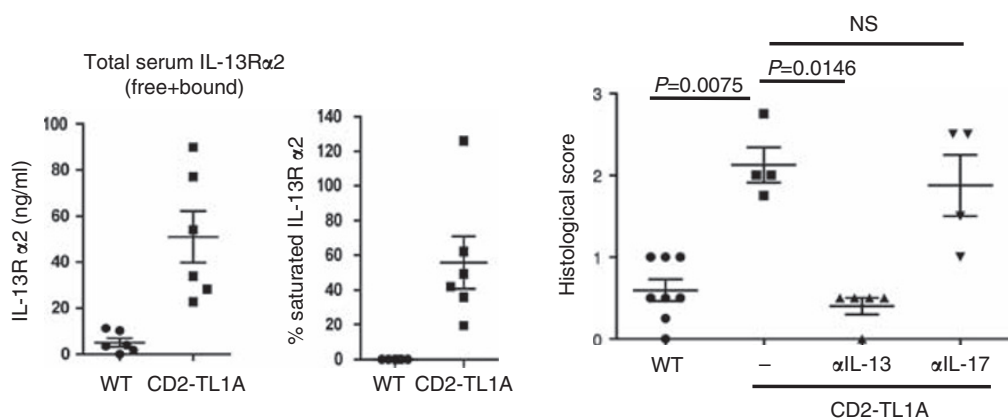

d
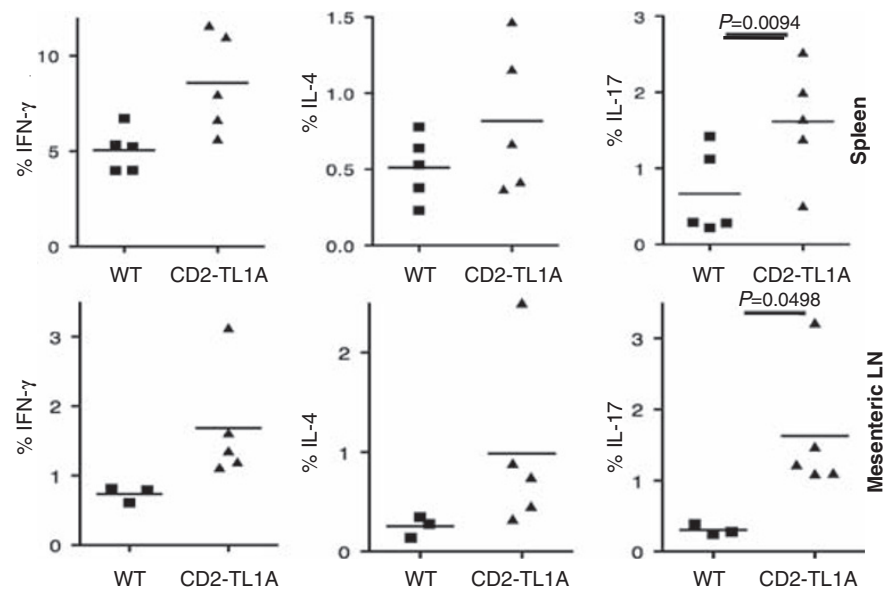

Figure 4 Cytokine expression in CD2-TL1A transgenic (Tg) mice. (a) mRNA expression of the indicated cytokines was analyzed from the indicated sections of intestine by quantitative reverse transcriptase-PCR (RT-PCR) from 8- to 52-week-old line R6 CD2-TL1ATg mice and age-matched controls. Gene expression was normalized first to $\beta 2$-microglobulin for each sample and then the average gene expression of the control mice for each region of intestine was normalized to 1.0. Statistical significance of comparisons of Tg vs. wild-type (WT) groups is indicated above (Mann-Whitney test). (b) Mast cell accumulation in the submucosa of tissue sections from CD2-TL1ATg mice. Toluidine blue staining of ileal sections of a 6-month-old mouse line R6 TL1ATg mouse and an age-matched control mouse is shown. (c) Soluble IL-13R $\alpha 2$ and the saturation of IL13R $\alpha 2$ in sera from CD2 TL1A transgenic mice (line R6) and controls. (d) Intracellular cytokine staining of T cells isolated from the indicated tissues of 8-10 weeks old CD2-TL1ATg or control mice stimulated with phorbol myristate acetate (PMA) and ionomycin. The percentage of CD4 ${ }^{+} \mathrm{T}$ cells expressing the indicated cytokine is shown, with the average of each group indicated by the line and significant differences between WT and Tg samples indicated (two-tailed Student's $t$-test). (e) Histological analysis of the ileum sections of intestine in CD2-TL1A transgenic mice treated with $10 \mathrm{mg} \mathrm{kg}^{-1}$ neutralizing antibodies against interleukin (IL)-13 or IL-17 intraperitoneally weekly for 6-8 weeks beginning at 2 weeks of age. Histological scores of non-transgenic littermates and littermates treated with isotype control antibody are shown (statistical analysis using Mann-Whitney test). Anti IL-13 antibody data are representative of two independent experiments. NS, not significant.

also reduced to background levels in TL1ATg $\times$ DR3KO mice (Figure 5c). In addition, the length of the small intestine from $\mathrm{TL} 1 \mathrm{ATg} \times \mathrm{DR} 3 \mathrm{KO}$ mice was significantly decreased compared with TL1ATg mice (Figure 5d). These results show that DR3 is the primary receptor mediating the effects of TL1A expressed constitutively in transgenic mice.

\section{NKT cell and FoxP3 ${ }^{+}$regulatory T-cell function in CD2-TL1A transgenic mice}

Inflammation in various models of IBD can result from dysfunction of natural killer T (NKT) cells or forkhead box P3 (FoxP3)-positive regulatory T cells (Tregs). ${ }^{21-24}$ We used CD1d tetramers loaded with the synthetic glycolipid PBS57 to detect invariant NKT (iNKT) expressing J $\alpha 18$ reactive against CD1d-restricted glycosphingolipids. ${ }^{25}$ Interestingly, although in some contexts these cells have been found to be proinflammatory, we observed reduced percentages and absolute numbers of iNKT cells in the spleen, liver, and lamina propria of TL1ATg mice compared with wild-type littermate controls (Supplementary Figure S6A, B online). This was likely due to peripheral depletion because percentages and numbers of iNKT cells in the thymus were less significantly reduced in the same mice. The iNKT cells can be positive or negative regulators of immune responses depending on the context. ${ }^{26,27}$ Although we cannot definitively rule out a pathogenic role for iNKT in this model until TL1A mice are back-crossed to J $\alpha 18$ deficient mice, these results suggest that depletion of iNKT is more likely secondary to the ongoing intestinal inflammation, as has been seen in human IBD and other diseases involving intestinal inflammation. ${ }^{28,29}$ 
a

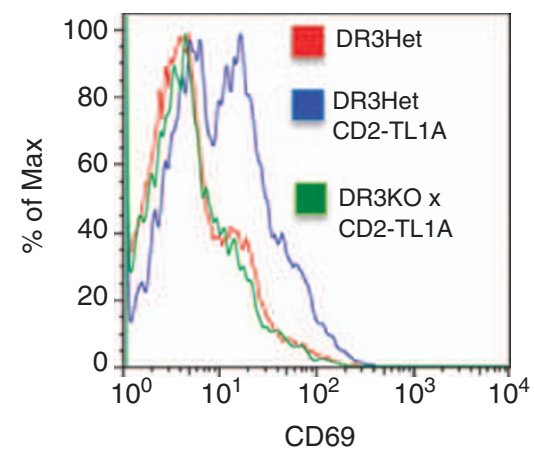

b

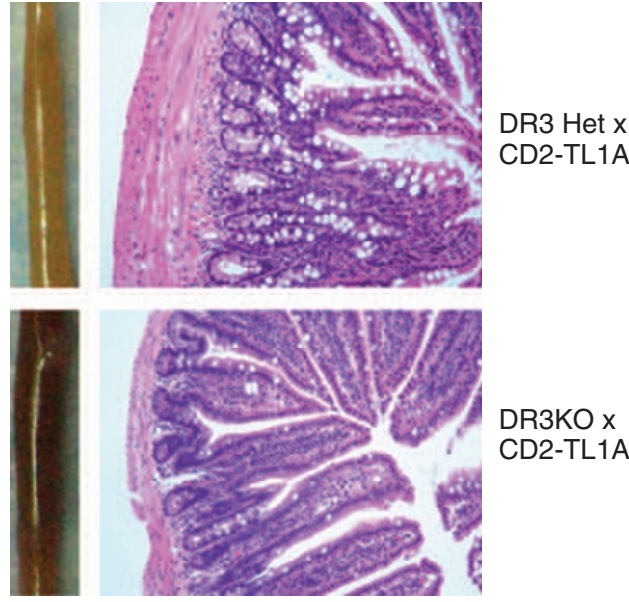

d
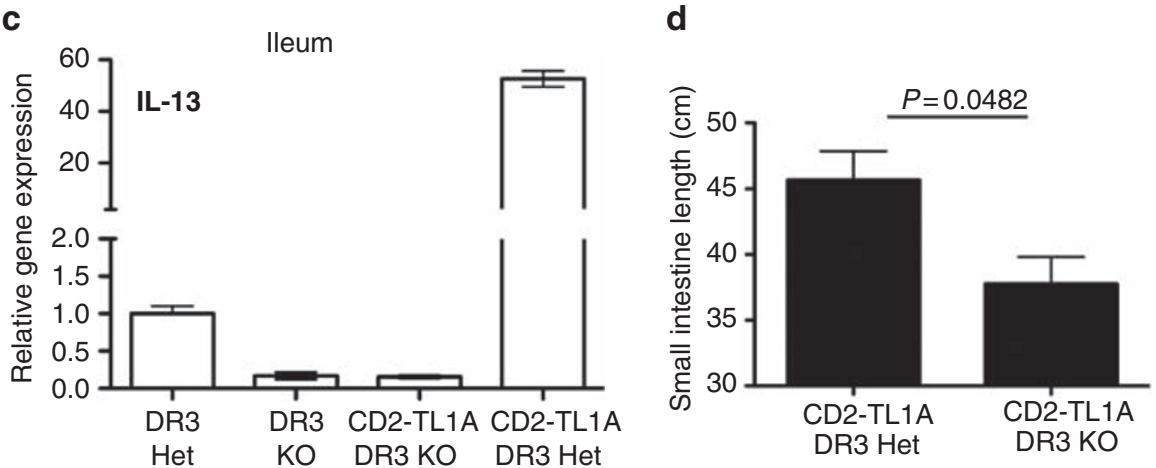

Figure 5 T-cell activation and intestinal inflammation in TL1A transgenic (Tg) mice depend on DR3. CD2-TL1A line R6 transgenic mice were crossed to DR3-deficient mice on a B6 background to generate DR3KO-TL1ATg mice. (a) CD69 expression on the surface of TCRb ${ }^{+} \mathrm{CD} 4{ }^{+} \mathrm{T}$ cells from the spleens of mice of the indicated genotypes of 20-24 weeks old mice. (b) Gross and histological appearance of representative ileum sections from mice of the indicated genotypes at 3 months of age. (c) Expression of IL-13 mRNA in the ileum of mice of the indicated genotypes at 8 weeks of age. Numbers are the average of triplicate measurements from 1 to 2 mice each. (d) Small intestine length of 8-20 weeks old DR3KO-TL1ATg $(n=3)$ and DR3Het CD2-TL1ATg mice ( $n=4)$. Statistical analysis using two-tailed unpaired Student's $t$-test.

In further studies, we focused on Treg numbers and function in CD2-TL1A transgenic mice. We first determined the percentage of $\mathrm{FoxP}^{+} \mathrm{CD} 4{ }^{+} \mathrm{T}$ cells in various tissues of $\mathrm{CD} 2-$ TL1A transgenic mice. We found that $\mathrm{CD} 4^{+} \mathrm{T}$ cells from mLNs and spleen were enriched in FoxP $3{ }^{+} \mathrm{CD} 4^{+} \mathrm{T}$ cells (Figure 6a). Lamina propria $\mathrm{T}$ cells were also enriched in FoxP3-expressing cells that, like Tregs from wild-type mice, expressed only low levels of CD25 (Figure 6b). To determine whether Tregs from CD2-TL1A transgenic mice exhibit normal regulatory function, we isolated FoxP $3{ }^{+} \mathrm{CD} 4{ }^{+}$cells from CD2-TL1A transgenic and control mice crossed to a knock-in mouse line expressing a FoxP3-green fluorescent protein (GFP) fusion protein. ${ }^{30}$ This enabled sorting of live FoxP3 ${ }^{+}$Tregs. Highly purified FACS (fluorescence-activated cell sorting)-sorted Tregs from TL1A transgenic mice were somewhat less effective than wild-type Tregs at suppressing proliferation of wild-type T cells (Figure 6c). When DR3-deficient responder T cells were used in these assays, proliferation was suppressed by TL1A transgenic Tregs similar to wild-type Tregs (Figure 6c). This suggests that TL1A expressed by transgenic Tregs in these cultures counteracts the proliferative suppression through the DR3 receptor on tresponder.
Antigen delivered to the small intestine can be presented by specialized DCs to generate antigen-specific Tregs in a manner dependent on transforming growth factor- $\beta$ (TGF- $\beta$ ) and retinoic acid. ${ }^{31,32}$ Although total Treg numbers are increased in TL1A transgenic mice, TL1A might suppress the production of antigen-specific Treg, which could allow escape of autoreactive $\mathrm{T}$ cells from peripheral tolerance. To test this idea, we activated naive FoxP3-negative T cells in the presence of TGF- $\beta$, which promotes the generation of inducible Tregs (iTregs) with a suppressive phenotype ${ }^{33}$ with or without TL1A. TL1A strongly suppressed induction of FoxP3expressing iTregs from naive precursors (Figure 6d). TL1A did not divert differentiation of T cells into IL-17 producers (Figure 6d). DR3 on T cells was necessary for this effect, as TL1A could not suppress iTreg generation in DR3-deficient $\mathrm{T}$ cells. In addition, DR3-deficient $\mathrm{T}$ cells differentiated into FoxP3-positive Tregs with enhanced efficiency, suggesting that endogenous TL1A-DR3 interactions suppress development of iTregs. These data identify two distinct defects in Treg generation and function in TL1A transgenic mice that may result in inefficient control of the excess effector $\mathrm{T}$ cells produced in these mice by Tregs. 
a
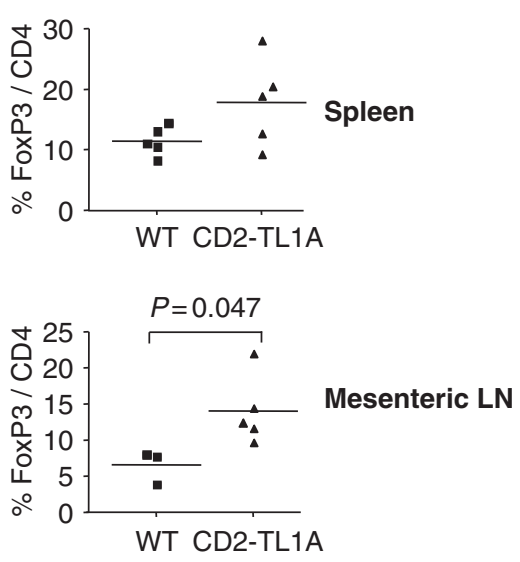

b

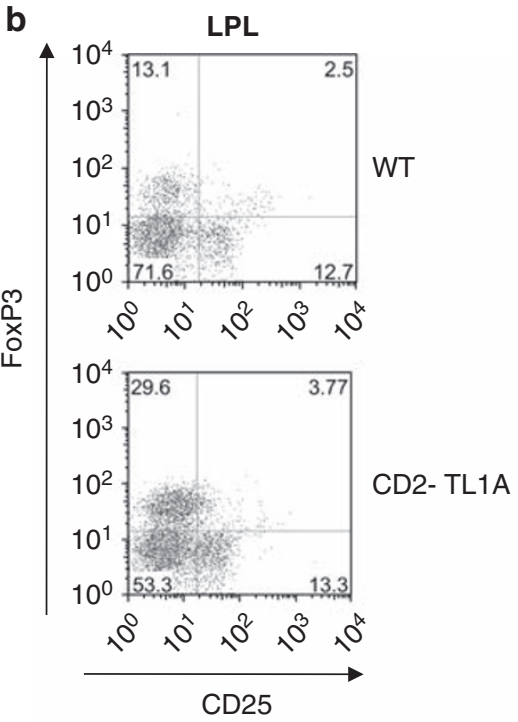

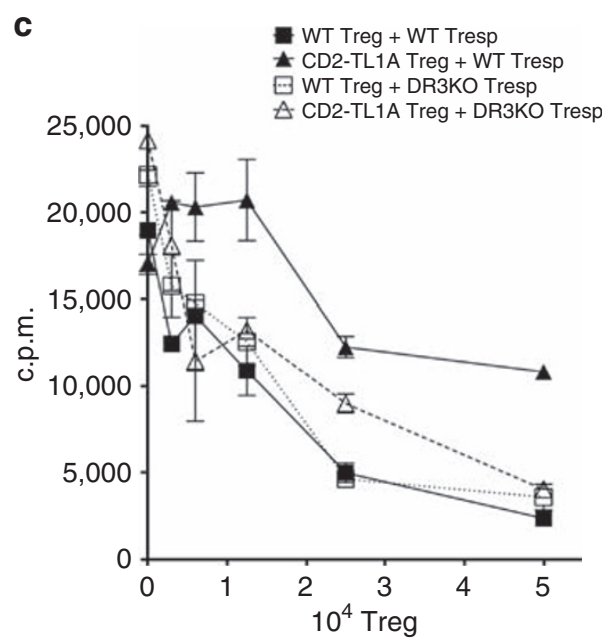

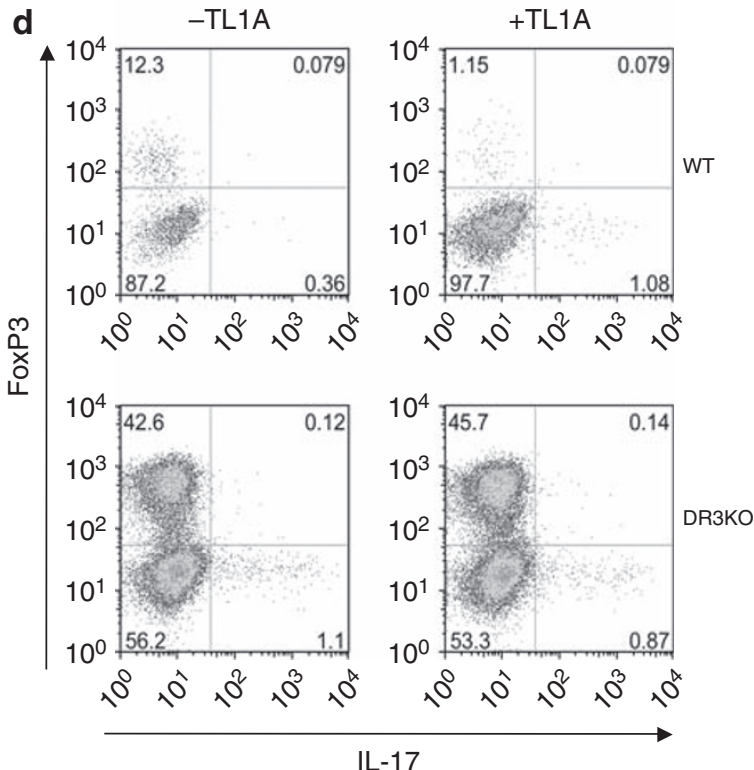

Figure 6 Effects of TL1A on the frequency, function, and generation of regulatory T cells. (a) The percentage of FoxP3-positive CD4 ${ }^{+} \mathrm{T}$ cells in the indicated tissues of 8-16 weeks old CD2-TL1A transgenic and control mice. The line represents the average percent expression. Statistical analysis using two-tailed unpaired Student's $t$-test. (b) FoxP3 and CD25 expression within CD4 ${ }^{+} \mathrm{T}$ cells isolated from the small intestinal lamina propria of representative CD2-TL1A transgenic mice and controls. (c) Regulatory T cell (Treg) suppressive assay with $5 \times 10^{4}$ naive C57BL/6 wild-type (WT) or DR3-deficient $\mathrm{CD}^{+} \mathrm{T}$ cells mixed with the indicated number of FoxP3 ${ }^{+}$Tregs from WT or TL1A transgenic mice. Proliferation assayed by ${ }^{3} \mathrm{H}$-thymidine incorporation is shown for triplicate. These data are representative of two independent experiments. (d) Polarization of naive CD4 ${ }^{+} \mathrm{T}$ cells of C57BL/6 WT or DR3-deficient mice toward inducible Tregs (iTregs) in the presence of WT antigen-presenting cells (APCs). Representative of two experiments using DR3KO mice and controls.

\section{Role of the T-cell repertoire in pathology driven by TL1A}

To determine whether the costimulation and intestinal inflammation triggered by TL1A is dependent on T cells capable of recognizing microflora or other environmental mucosal antigens, we restricted the T-cell repertoire of TL1A transgenic mice to a monoclonal specificity by back-crossing the R6 CD2-TL1A transgenic line to the OT-II ovalbumin-specific TCR transgene on a recombination activation gene-1 (RAG1)-deficient background. All the T cells in these mice express the OT-II TCR specific for ovalbumin, with little or no cross-reactivity to endogenous antigens. On this background, transgenic TL1A expression was not able to increase the percentage of $\mathrm{CD} 69^{+} \mathrm{T}$ cells or memory T cells, and all T cells in either TL1A transgenic or control mice were of a $\mathrm{CD} 4{ }^{+}$naive phenotype (Figure 7a). Inspection of the small intestine of OT-II Tg-Rag $1^{-/-}$mice with or without the TL1A transgene revealed a significant amelioration of TL1A-dependent intestinal changes by the OT-II/Rag1 ${ }^{-1-}$ background. As shown in the examples of ileal sections in Figure $7 \mathbf{b}$, thickening of the submucosa and muscularis and distortion of the villi are 
partially reversed in OT-II Tg-Rag1 ${ }^{-1-}$ mice compared with parental CD2-TL1ATg samples. Analysis of inflammatory changes in ileum histology in a cohort of TL1ATg $\times$ OT-II $\mathrm{Tg}$ mice on the RAG1 background revealed only nonsignificant differences from control OT-II Tg-RAG1-deficient mice (Figure 7c). However, the small intestine was still lengthened in the TL1A transgenic mice compared with controls (Figure 7d). Analysis of cytokine mRNA in intestinal tissue from these mice revealed generally lower levels of IL-13 compared with CD2-TL1ATg mice, but remained significantly higher than in OT-II Tg-Rag1 ${ }^{-/-}$controls in jejunum and colon (Figure 7e). Both IL-13 and IL-17 mRNA levels actually increased in the colon compared with TL1A transgenic mice with an intact lymphocyte compartment, which may be because of the absence of Tregs in these mice. Quantitation of goblet cell number and size in the ileum of OT-II TCR transgenic mice revealed that there was still significant elevation in the TL1A transgenic samples on the OT-II RAG1 ${ }^{-/-}$background (Figure 7f). These data suggest that in the absence of recognition of endogenous antigens by the TCR, constitutive expression of TL1A is not able to costimulate $\mathrm{T}$ cells, abrogating the accumulation of activated T cells. However, IL-13 production and goblet cell hyperplasia are still stimulated by TL1A in a manner independent of a full TCR repertoire.

\section{TNBS colitis depends on TL1A-DR3 interactions}

Prior studies have shown that inhibition of TL1A-DR3 interactions by administration of blocking anti-TL1A antibody has a modestly ameliorating effect on dextran sodium sulfate (DSS)-induced colonic inflammation. ${ }^{34}$ Similarly, as shown here, DR3-deficient mice are modestly protected from this model of intestinal inflammation at early time points after colitis induction (Supplementary Figure S7 online). T celldeficient mice remain susceptible to DSS colitis, indicating that this model is mainly because of an innate immune response to intestinal flora. ${ }^{35,36}$ To determine whether TL1A-DR3 interactions are necessary in an inducible $\mathrm{T}$ cell-dependent murine model of colitis, we turned to the 2,4,6 trinitrobenzenesulfonic acid (TNBS) model of colitis, which depends on T cells secreting IFN- $\gamma$ in the acute phase. ${ }^{13}$ We induced acute TNBS colitis in susceptible C57BL/10 mice with and without concomitant intraperitoneal injection of the antagonistic antiTL1A mAb 5.4G6 (Supplementary Figure S1 online), DR3-Fc, or an antagonistic Fab mAb to DR3. Mice treated with antiTL1A and to a lesser extent with DR3-Fc or anti-DR3 Fab were remarkably protected from the weight loss and mortality in TNBS-induced colitis (Figure 8a-c). Reduced morbidity and mortality correlated with dramatically reduced microscopic inflammation (Figure 8d). This protection from TNBS colitis did not stem from changes in the local Treg population, as the percentage of FoxP3-expressing $\mathrm{T}$ cells in the colon was slightly decreased in TNBS colitis but not different in any of the treatment groups (Figure 8e). Taken together, these results show a critical role for TL1A-DR3 interactions in this inducible model of colitis that depends on T cells, but is associated with a different anatomical location of inflammation and a contrasting set of cytokines than that seen in the setting of chronic TL1A overexpression.

\section{DISCUSSION}

These results establish that constitutive expression of the TNFfamily cytokine TL1A leads to dose-dependent gastrointestinal inflammation marked by continuous epithelial and goblet cell hyperplasia, thickening of the submucosal layers of the intestine, and lamina propria inflammatory infiltrates. All these changes are confined to the small bowel and are most intense in the terminal ileum. The pathology induced by TL1A differed significantly from murine models of small bowel inflammation associated with overproduction of TNF ${ }^{37,38}$ or TNFSF $14 .{ }^{39}$ In TL1A-driven inflammation, IL-13 was uniquely important for the pathogenesis of intestinal inflammation, as shown by significant amelioration of the pathology by treatment with anti-IL13 $\mathrm{mAb}$. Although IL-17 production was also modestly increased in mLNs and lamina propria cells in CD2-TL1A transgenic mice, the pattern of increased IL-17 expression in various segments of the small intestine did not correlate with areas of maximum inflammation, and blocking IL-17 had little effect on the changes in the small intestine induced by TL1A, suggesting that IL-17 has a secondary role in the pathogenesis of intestinal inflammation driven by TL1A.

Much of the intestinal pathology observed in TL1A transgenic mice may be attributed to IL-13, as this cytokine can act directly on epithelial cells to cause goblet cell hyperplasia and mucous production in both the lung and intestine. ${ }^{40,41}$ Smooth muscle cells can also respond to IL-13 with hypertrophy and increased contractility ${ }^{42}$ suggesting that the hypertrophy of the muscularis layer of the small intestine seen in TL1A transgenic mice may also be because of IL-13. The colon may be spared from IL-13-driven pathology because of elevated levels of nonsignaling IL-13R2 receptor subunit. ${ }^{43}$ The lack of IFN- $\gamma$ production that we observed in TL1A transgenic mice may explain the relative longevity of these mice despite the inflammatory pathology observed, as IFN- $\gamma$ is associated with enterocyte cell death that may lead to more severe clinical consequences. ${ }^{44}$ Overall, the pathology induced by constitutive TL1A expression bears a striking resemblance to the intestinal response to nematode infections, where IL-13 mediates induction of goblet cell hyperplasia, mucous production, and muscular hypertrophy that aids in worm expulsion, but spares the host from lethal inflammation. ${ }^{41,45,46}$ The similarity between the pathology caused by the constitutive expression of TL1A and nematode infection raises the interesting possibility that TL1A might be an important inducer of IL-13 during such infections.

These data establish a novel link between TL1A, acting through its receptor DR3, to induce IL-13 and associated gastrointestinal pathology. Previous studies have shown that TL1ADR3 interactions can enhance secretion of multiple cytokines, not just those associated with Th2 immune responses. IL-13 may be produced in CD2-TL1A transgenic mice by many cell types, including T cells, iNKT cells, intraepithelial intestinal NK cells, mast cells, and basophils. ${ }^{41,47,48}$ As DR3 is mainly expressed on lymphocytes, T cells, NKT cells, and NK cells, these cells are 
a

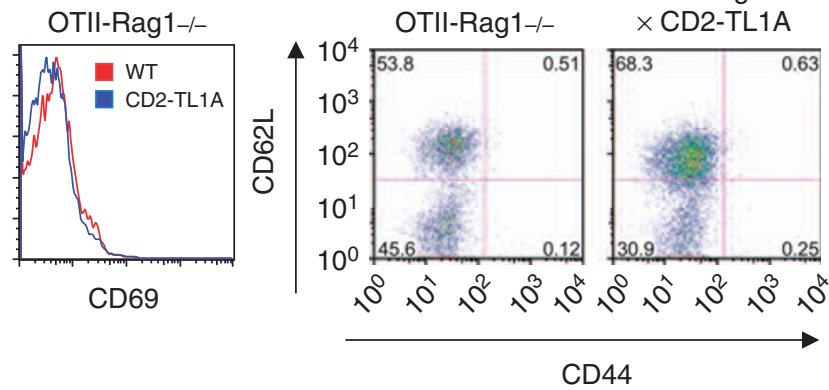

b

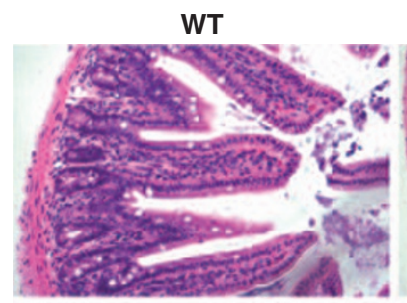

OTII Rag1-/-

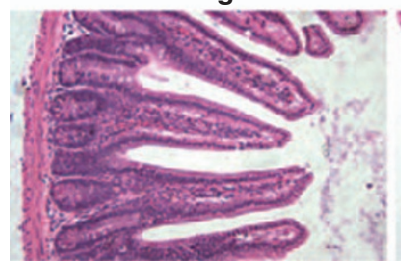

C

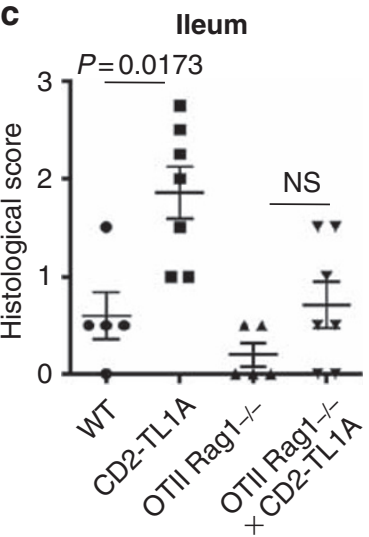

CD44

CD2-TL1A

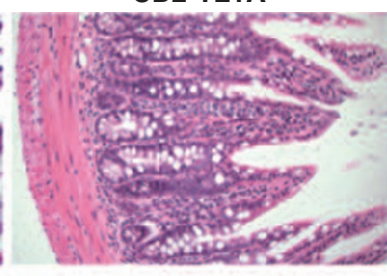

OTII Rag1-I- × CD2-TL1A

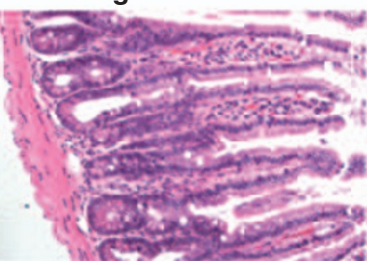

e

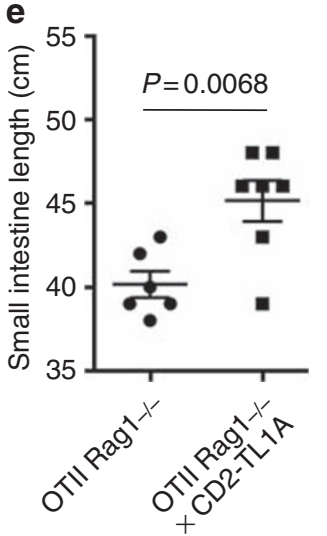

d
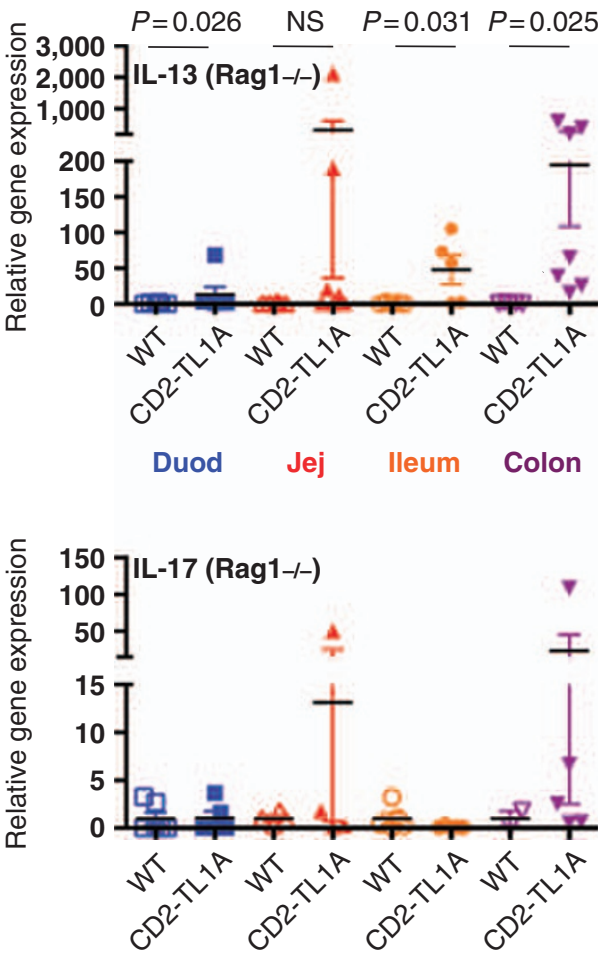

Duod Jej lleum Colon f

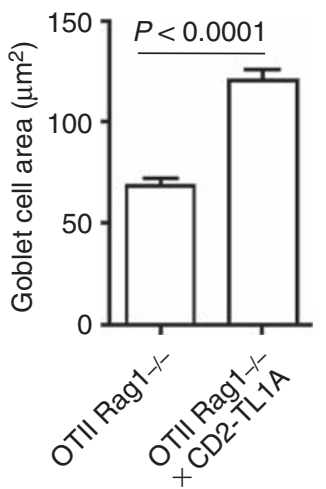

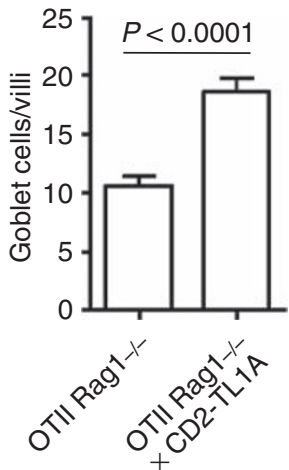

Figure 7 Restriction of the T-cell repertoire abolishes peripheral T-cell hyperactivation but does not prevent intestinal inflammation in the presence of transgenic (Tg) TL1A. (a) CD69 expression and CD44/CD62L surface expression in splenic CD4+ $\mathrm{T}$ cells from 20-24 weeks old TL1A transgenic and control mice on an OT-II TCR, Rag1-/- background. (b) Representative tissue sections from the ileum of TL1A transgenic and control mice on an OT-II TCR Rag1-/- background, shown with CD2-TL1ATg and wild-type (WT) controls on a C57BL/6 background. (c) Histological scores of ileal sections from a cohort of OT-II TCR Rag1-deficient mice with and without expression of the TL1A transgene and age-matched WT and TL1A transgenic controls from 3 to 6 months of age. Statistical analysis using Mann-Whitney test. (d) IL-13 and IL-17 mRNA expression in the indicated sections of intestine from TL1A transgenic and control mice on an OT-II TCR Rag $1^{-/-}$background. Gene expression is normalized to the average of WT mice and means and s.e.m. are indicated by the horizontal bars. The $P$-values from significant differences using Mann-Whitney test are given. (e) Small intestinal length from the cohort of mice analyzed in c. Statistical analysis using two-tailed unpaired Student's $t$-test. (f) Average goblet cell area and goblet cells per villus calculated from analysis of five sections per genotype as in Figure 3. Statistical analysis using two-tailed unpaired Student's $t$-test.

among likely cellular sources of IL-13. However, intracellular staining of T cells in the mLNs and intestinal lamina propria only revealed small increases in Th2 cells, suggesting that other cell types may contribute to IL-13 production induced by TL1A. NKT cells have been found to produce IL-13 and are implicated in IBD, particularly in ulcerative colitis. ${ }^{22}$ However as these cells are relatively depleted in TL1A transgenic mice, they are less likely to be required. Intraepithelial NK cells or recently identified novel "innate" lymphocyte subsets in the mLNs and fatassociated lymphoid clusters that produce IL-13 in the setting of helminth infections ${ }^{48-50}$ are other possible sources of IL-13 produced in response to TL1A. 

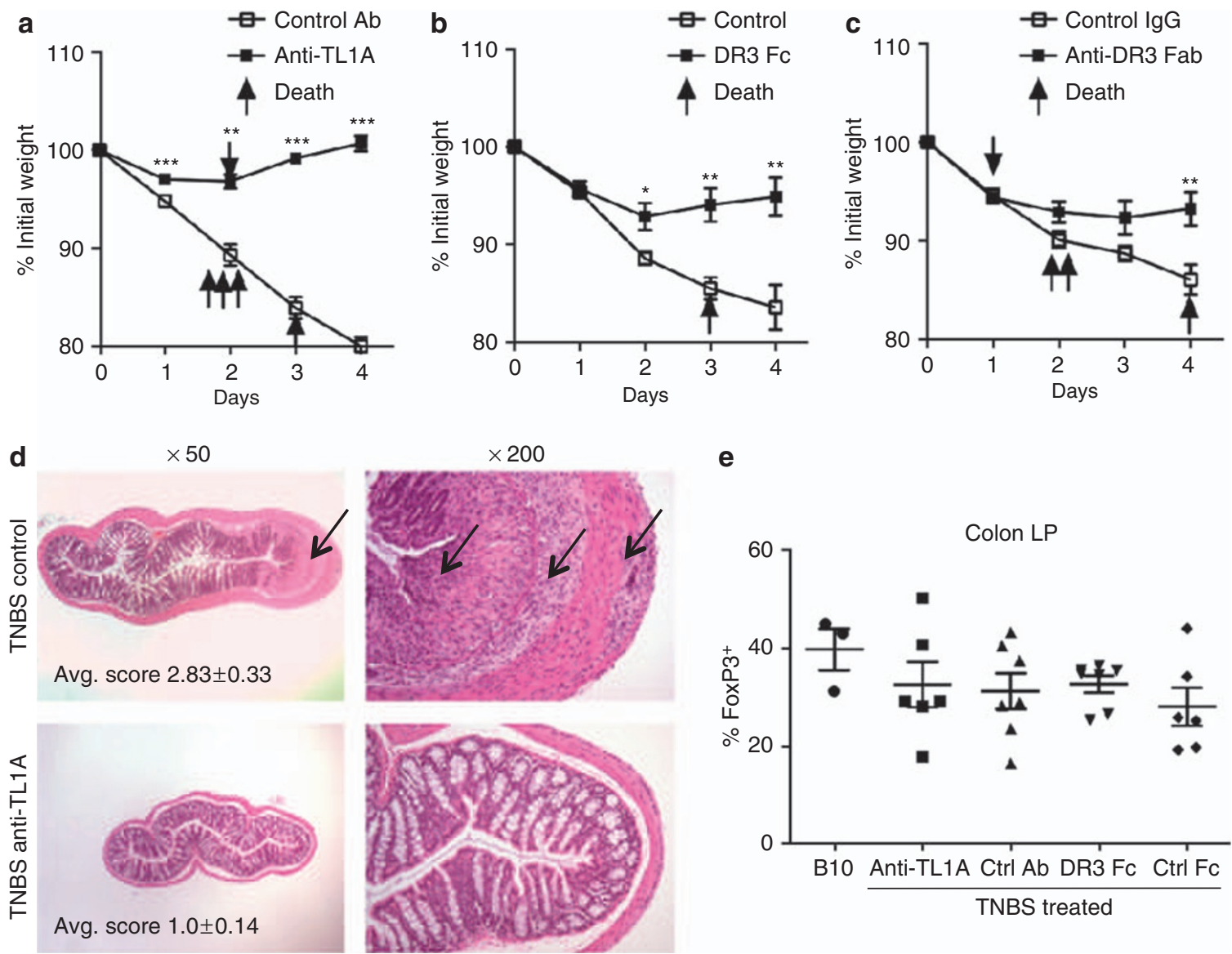

e

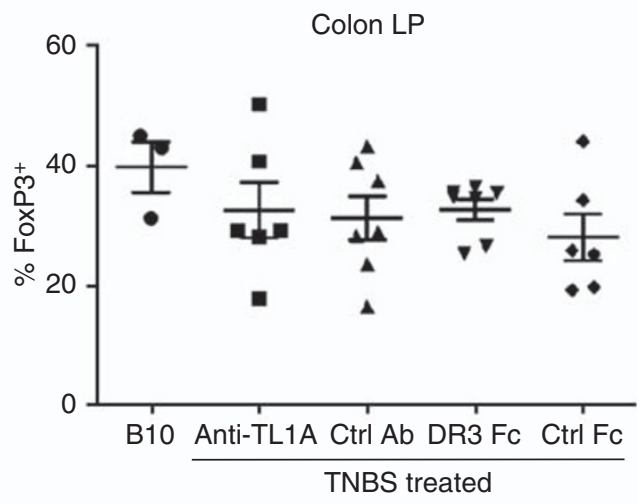

Figure 8 Dependence of 2,4,6 trinitrobenzenesulfonic acid (TNBS)-induced colitis on TL1A. (a) Weight loss in a cohort of mice induced to develop TNBS colitis with $20 \mathrm{mg} \mathrm{kg}^{-1}$ anti-TL1A (open squares, $n=10$ ) or control hamster Ig (closed squares, $n=10$ ) injected intraperitoneally (IP) on days -1 and 0 . Each point represents the average weight of the cohort. Mice that died before the end of the experiment are indicated with arrows. Anti-TL1A data are representative of two independent experiments with a minimum of eight mice per group. ${ }^{* *} P<0.01,{ }^{* * *} P<0.0001$ by unpaired two-tailed Student's $t$-test. (b) TNBS colitis experiment carried out as in a with DR3 Fc (closed squares, $n=8$ ) and control Fc (open squares, $n=8$ ). ${ }^{*} P<0.05$, ${ }^{\star *} P<0.01$ by unpaired two-tailed Student's $t$-test. (c) TNBS colitis experiment carried out as in a with anti-DR3 Fab (closed squares, $\left.n=10\right)$ and control lgG (open squares, $n=10$ ). ${ }^{*} P<0.01$ by unpaired two-tailed Student's $t$-test. (d) Representative hematoxylin and eosin (H\&E) sections of the colon from mice induced to develop TNBS colitis treated with control or anti-TL1A monoclonal antibody (mAb) as in a. Arrow indicates area of severe inflammation of the control Ab-treated mouse. Left panels are original magnification $\times 50$ and right panels original magnification $\times 200$ of the same sections. Average pathology scores of the mice in a at day 6 after induction of colitis are indicated. (e) FoxP3 $3^{+}$cells within $\mathrm{CD} 4^{+} \mathrm{T}$ cells isolated from colon lamina propria of mice induced to develop TNBS colitis treated with the indicated reagents.

$\mathrm{CD} 4{ }^{+} \mathrm{T}$ cells are activated and expanded in TL1A transgenic mice and this is abrogated when the T-cell repertoire is fixed through crossing these mice to the OT-II TCR transgenic mice on a Rag-deficient background. These results strongly suggest that TL1A-driven T-cell activation requires a concomitant TCR signal that is provided in vivo by antigens in the bowel lumen or other environmental antigens. Autoreactive $\mathrm{T}$ cells appear to be directly or indirectly required for some of the intestinal pathology in TL1A transgenic mice, because of the reduced histological scores in TL1A transgenic mice crossed to the OT-II TCR transgenic mice on a RAG1-deficient background. However, intestinal IL-13 and goblet cell hyperplasia are still present in TL1A transgenic mice on the OT-II/Rag1 ${ }^{-1-}$ background, suggesting that the TL1A present on resting $\mathrm{T}$ cells is sufficient to drive these pathological changes, likely acting through another cell type present in the small intestine.
Given the important role of Tregs in other models of intestinal inflammation, we examined Treg function in CD2-TL1A transgenic mice. Along with V. Taraban et al. (personal communication), we noted that Tregs are numerically increased in mice constitutively expressing TL1A driven either by CD2 or CD11c promoters. Tregs isolated from CD2-TL1A transgenic mice that constitutively produce TL1A are less effective at suppressing proliferation of naive $\mathrm{T}$ cells than wild-type Tregs, and V. Taraban et al. (personal communication) show that soluble recombinant TL1A can also partially reverse proliferative suppression by Tregs. This suggests that costimulation through DR3 can reverse suppression by Tregs, either directly, or indirectly through promoting cytokine production. This is consistent with the known costimulatory effect of TL1A on Tcell proliferation that is mediated through DR3. Costimulation by DR3 is associated with increased production of multiple cytokines, including IL-2, ${ }^{3,4}$ and the ability of IL-2 to reverse 
suppression by Tregs. ${ }^{51}$ Recent studies have shown that orally delivered antigen can induce de novo generation of antigen-specific Tregs through antigen presentation by a specialized population of DCs capable of secreting retinoic acid and TGF- $\beta .^{31,52}$ We found that TL1A greatly inhibited TGF- $\beta$-induced differentiation of FoxP3 ${ }^{+} \mathrm{T}$ cells in vitro from naive T-cell precursors. This suggests that peripheral induction of Tregs in the gastrointestinal tract of transgenic mice might be defective in the setting of TL1A overexpression. It is likely that in TL1A transgenic mice, a combination of Treg defects and TL1Amediated costimulation of effector $\mathrm{T}$ cells results in failure of immunological tolerance to mucosal antigens, spontaneous T-cell activation, and intestinal inflammation.

The efficacy of blocking TL1A-DR3 interactions in the TNBS colitis model suggests that these interactions are necessary for IBD, involving a broader range of cytokines than is seen in TL1A overexpression. Antibody blockade of DR3-TL1A interactions by anti-TL1A, DR3-Fc, and an antagonistic anti-DR3 Fab all led to a striking and virtually complete inhibition of TNBS-induced colitis. In the acute phase, TNBS-induced colitis is associated with T cells secreting IFN- $\gamma,{ }^{53,54}$ and can be blocked by antibodies against the p40 common subunit of IL-12 and IL-23. ${ }^{55}$ More recently, IL-17 has also been implicated in the pathogenesis of acute TNBS colitis. ${ }^{56}$ Crohn's disease has been associated with IL-12 and IL-23, and ulcerative colitis with IL-13, ${ }^{57,58}$ and TL1A-DR3 interactions may contribute to IBD through either of these two pathways. Because no major defects in systemic immunity have thus far been found in DR3- or TL1A-deficient mice, treatment of IBD by blocking TL1A-DR3 interactions may have a favorable therapeutic index.

\section{METHODS}

Mice. DR3-deficient mice were obtained from Eddie C-Y Wang (University of Cardiff, UK) and FoxP3-GFP mice were obtained from Yasmine Belkaid (NIAID, NIH, Bethesda, MD). C57BL/6 and C57BL/10 mice were purchased from Jackson Laboratories (Bar Harbor, ME). OT-II TCR transgenic Rag1-deficient mice were obtained from the NIAID Taconic mouse contract facility. TL1A coding sequences were amplified by PCR with primers encoding a $5^{\prime}$ hemagglutin tag and cloned into the EcoR1 sites of a CD2 promoter/enhancer transgenic cassette vector, ${ }^{12}$ and a CD11c promoter/enhancer cassette vector. ${ }^{59}$ Transgenic mice were screened by southern blotting and subsequent generations screened by PCR using the following primers: hemagglutin forward 5' -CCATACGACGTCCCAGACTACGC-3' and TL1A reverse 5' -CAGGTGTCTCTCGGCTTGCC-3'. All animals were used under protocols approved by the NIAMS and NIAID animal care and use committee.

Measurement of gene expression by quantitative reverse transcriptase-PCR. Quantitative reverse transcriptase-PCR was performed using ABI PRISM 7700 (PE Applied Biosystems, Foster City, CA) sequence-detection system with qScript One-Step qRT-PCR Kit, Low ROX (Quanta BioSciences, Gaithersburg, MD). Predesigned primer/probe sets were from Applied Biosystems (PE Applied Biosystems, Foster City, CA): IFN- $\gamma$ (Mm00801778_m1), IL-13 (Mm00434204_m1), IL-17 (Mm00439619_m1), and $\beta 2$-microglobulin (Mm00437762_m1), and sequences designed to detect full-length TL1A were forward: 5' -CCCCGGAAAAGACTGTATGC-3'; reverse: 5'-GGTGAGTAAACTTGCTGTGGTGAA-3'; probe: 5' -TCGGG CCATAACAGAAGAGAGATCTGAGC-3'). Each measurement was normalized to expression of $\beta 2$-microglobulin $(\Delta \mathrm{Ct}) .2^{-\Delta \mathrm{Ct}}$ was then used as the level of gene expression. Gene expression levels were normalized to the average gene expression in control mice in each experiment.

\section{Detection of free and IL-13-complexed soluble IL-13R $\alpha 2$}

Serum levels of the IL-13/sIL-13R $\alpha 2$ complex were measured as previously described ${ }^{17}$ by enzyme-linked immunosorbent assay using affinity purified goat anti-mouse $\mathrm{Ab}$ ( $\mathrm{R} \& \mathrm{D}$ Systems) to capture the complex onto an Immulon $2-\mathrm{HB}$ microtiter plate, followed by detection with biotinlabeled anti-IL-13 mAb (courtesy of Centocor, Horsham, PA). Total levels (free and complexed) of sIL-13R $\alpha 2$ were detected with the same assay except that rIL-13 $\left(100 \mathrm{ng} \mathrm{ml}^{-1}\right)$ was added to the serum before performing the assay. The percent saturation of sIL-13R $\alpha 2$ with IL-13 was determined by dividing the concentration of IL-13/sIL-13R $\alpha 2$ complex by the total sIL-13R $\alpha 2$ concentration detected when rIL-13 was added to "pull down" free (non-complexed) sIL-13R $\alpha 2$.

Antibody treatments and generation of anti-TL1A, DR3-Fc, and antiDR3-Fab. Anti-TL1A mAbs were generated by immunizing Armenian hamsters with recombinant murine TL1A. Antibodies were screened primarily by flow cytometry against $293 \mathrm{~T}$ cells transfected with the $\mathrm{N}$-terminal GFP-TL1A fusion protein as described in Supplementary Figure S1 online. The mouse DR3-Fc protein consists of the extracellular domain of mouse DR3 (M1-Q197) that was cloned and fused with human Fc region at C-terminal generated by Human Genome Sciences (Rockville, MD). Anti-DR3 Fab was generated using mouse DR3-Fc to select ScFv from human naive phage display libraries (Cambridge Antibody Technology/MedImmune, Cambridge, UK). The specific binders were further screened for their ability to inhibit the binding activity of mouse TL1A to DR3-Fc. The selected clone was sequenced and subcloned into a Fab expression vector where mouse serum albumin sequence was fused to the $\mathrm{C}$-terminal of $\mathrm{CH} 1$ to generate $\mathrm{mDR} 3-\mathrm{Fab}$ mouse serum albumin. The fusion protein was expressed in 293 cells and purified through affinity chromatography by Human Genome Sciences. Anti IL-17 M210 mAb (mouse IgG2a) was obtained from Dr Joel Tocker (Amgen, Thousand Oaks, CA) and anti IL-13 mAb (ratIgG1) was obtained from Centocor/ Johnson and Johnson (Horsham, PA).

T-cell polarization, flow cytometry, and measurement of intracellular cytokine expression. For polarization studies, $5 \times 10^{5} \mathrm{~T}$ cell-depleted antigen-presenting cells were cultured with $10^{5}$ naive $\mathrm{CD} 4^{+} \mathrm{T}$ cells from C57BL/6 mice or DR3-deficient mice. iTreg cell polarization was driven with rhTGF- $\beta$ (10 $\left.\mathrm{ng} \mathrm{m}^{-1}\right)$ (eBioscience, San Diego, CA) and rhIL-2 (100 U) in the presence of $5 \mu \mathrm{g} \mathrm{ml}^{-1}$ soluble anti-CD3/anti-CD28. After 3 days of culture, intracellular cytokine staining was performed as described below. All antibodies for flow cytometry were purchased from eBioscience unless indicated otherwise. CD1d/PBS57 tetramers that recognize V $\alpha 14$ iNKT cells were prepared by the National Institute of Health (NIH) tetramer core facility. Preparation of lamina propria lymphocytes was carried out as previously described. ${ }^{60}$ For measurement of intracellular cytokine production, T cells were restimulated either for $5 \mathrm{~h}$ for splenocytes or $\mathrm{mLNs}$ or for $2 \mathrm{~h}$ for lipoprotein lipase with $10 \mathrm{~nm}$ phorbol myristate acetate and $1 \mu \mathrm{M}$ ionomycin in the presence of monensin. Cells were fixed in 3\% paraformaldheyde, permeabilized in $1 \%$ saponin, and analyzed on a FACS Calibur flow cytometer (Becton Dickinson, Franklin Lakes, NJ). For detection of FoxP3 expression, fixation and permeabilization solutions provided by the manufacturer (eBioscience) were used.

Immunofluorescence. Sections of intestine were frozen in optimal cutting temperature solution. Samples were sectioned and stained with anti-E-cadherin-fluorescein isothiocyanate, anti-CD4-PECy5, and 4,6diamidino-2-phenylindole followed by examination under a fluorescence microscope. 
T-cell suppression assay. $\mathrm{CD} 4{ }^{+} \mathrm{CD} 25^{-}$responder T cells $\left(5 \times 10^{4}\right)$ with irradiated T-depleted antigen-presenting cells $\left(5 \times 10^{4}\right)$ and various concentrations of Tregs (FoxP3-GFP ${ }^{+} \mathrm{CD} 4{ }^{+} \mathrm{CD} 25^{+}$) were cultured in the presence of $0.125 \mu \mathrm{g}$ anti-CD3. After 3 days, $1 \mu \mathrm{Ci}$ of $3 \mathrm{H}$-thymidin was added for the last $16 \mathrm{~h}$ of culture.

TNBS- and DSS-induced colitis. TNBS colitis was induced by intrarectal administration of TNBS in B10 mice as previously described. ${ }^{13}$ In addition, $20 \mathrm{mg} \mathrm{kg}^{-1}$ anti-TL1A, DR3-Fc protein, anti-DR3 Fab, or control hamster IgG, control Fc protein, or control mouse IgG, respectively, was administered intraperitoneally on days -1 and 0 . Mice were weighed every day for 5 days and then colon were harvested, cut into $1 \mathrm{~cm}$ pieces, and fixed in 10\% neutral-buffered formalin (Sigma, St Louis, MO). Mice losing $>20 \%$ body weight were killed according to the guidelines of the animal care and use committee. Samples were embedded, sectioned, and stained with hematoxylin and eosin. Each section was scored in a blind fashion according to previously described criteria. ${ }^{13}$ DSS colitis was induced by feeding mice with $5 \%(\mathrm{w} / \mathrm{v})$ DSS in their drinking water for 5 days, followed by 2 days of consumption of water. Mice were weighed every day for 7 days. Inflammatory changes were scored on samples masked to treatment or genotype according to a scoring system adapted from that used in TNBS colitis. ${ }^{13}$

SUPPLEMENTARY MATERIAL is linked to the online version of the paper at http://www.nature.com/mi

\section{ACKNOWLEDGMENTS}

We thank Anthony Cruz for technical assistance, Jin-Moo Lee for providing the TL1A cDNA, Pat Korty and Ethan Shevach for assistance with generating monoclonal antibodies, Lionel Feigenbaum and the $\mathrm{NCl}$ transgenic facility for producing the mice, Brian Kelsall and Yasmine Belkaid for reagents and discussions, Eddie C-Y Wang for DR3-deficient mice, Anuk Das for providing the anti-mouse IL-13 mAb, Joel Tocker for providing the anti-mouse IL-17 mAb, Pascal Schneider for ACRP-TL1A fusion protein, and Jim Simone and the NIAMS flow cytometry facility for cell sorting. This work was supported by NIAMS intramural research funding and a fellowship to F.M. from the Crohn's and Colitis Foundation of America.

\section{DISCLOSURE}

Thi-Sau Migone and Larry Lo are employees of Human Genome Sciences, and owners of HGSI stock. The remaining authors declared no conflict of interest.

() 2011 Society for Mucosal Immunology

\section{REFERENCES}

1. Screaton, G.R. et al. LARD: a new lymphoid-specific death domain containing receptor regulated by alternative pre-mRNA splicing. Proc. Natl. Acad. Sci. USA 94, 4615-4619 (1997).

2. Marsters, S.A. et al. Apo-3, a new member of the tumor necrosis factor receptor family, contains a death domain and activates apoptosis and NF-kappa B. Curr. Biol. 6, 1669-1676 (1996).

3. Migone, T.S. et al. TL1A is a TNF-like ligand for DR3 and TR6/DcR3 and functions as a T cell costimulator. Immunity 16, 479-492 (2002).

4. Meylan, F. et al. The TNF-family receptor DR3 is essential for diverse T cell-mediated inflammatory diseases. Immunity 29, 79-89 (2008).

5. Pappu, B.P. et al. TL1A-DR3 interaction regulates Th17 cell function and Th17-mediated autoimmune disease. J. Exp. Med. 205, 1049-1062 (2008).

6. Fang, L., Adkins, B., Deyev, V. \& Podack, E.R. Essential role of TNF receptor superfamily 25 (TNFRSF25) in the development of allergic lung inflammation. J. Exp. Med. 205, 1037-1048 (2008).

7. Prehn, J.L. et al. Potential role for TL1A, the new TNF-family member and potent costimulator of IFN-gamma, in mucosal inflammation. Clin. Immunol. 112, 66-77 (2004).

8. Bamias, G. et al. Role of TL1A and its receptor DR3 in two models of chronic murine ileitis. Proc. Natl. Acad. Sci. USA 103, 8441-84416 (2006).

9. Thiebaut, R. et al. TNFSF15 polymorphisms are associated with susceptibility to inflammatory bowel disease in a new European cohort. Am. J. Gastroenterol. 104, 384-391 (2009).
10. Yamazaki, K. et al. Single nucleotide polymorphisms in TNFSF15 confer susceptibility to Crohn's disease. Hum. Mol. Genet. 14, 3499-3506 (2005).

11. Barrett, J.C. et al. Genome-wide association defines more than 30 distinct susceptibility loci for Crohn's disease. Nat. Genet. 40, 955-962 (2008).

12. Zhumabekov, T., Corbella, P., Tolaini, M. \& Kioussis, D. Improved version of a human CD2 minigene based vector for T cell- specific expression in transgenic mice. J. Immunol. Methods 185, 133-140 (1995).

13. Scheiffele, F. \& Fuss, I. Induction of TNBS colitis in mice. In Current Protocols in Immunology (Coligan, J.E., Bierer, B.E., Margulies, D.H., Shevach, E.M., Strober, W. eds) (Wiley-Blackwell, Hoboken, NJ, (Unit 15.19.1-15.19.14), 2002).

14. Cassatella, M.A. et al. Soluble TNF-like cytokine (TL1A) production by immune complexes stimulated monocytes in rheumatoid arthritis. J. Immunol. 178, 7325-7333 (2007).

15. Prehn, J.L., Thomas, L.S., Landers, C.J., Yu, Q.T., Michelsen, K.S. \& Targan, S.R. The T cell costimulator TL1A is induced by FcgammaR signaling in human monocytes and dendritic cells. J. Immunol. 178, 4033-4038 (2007).

16. Brocker, T., Riedinger, M. \& Karjalainen, K. Driving gene expression specifically in dendritic cells. Adv. Exp. Med. Biol. 417, 55-57 (1997).

17. Khodoun, M. et al. Differences in expression, affinity, and function of soluble (s)|L-4Ralpha and sIL-13Ralpha2 suggest opposite effects on allergic responses. J. Immunol. 179, 6429-6438 (2007).

18. Annacker, O. et al. Essential role for CD103 in the T cell-mediated regulation of experimental colitis. J. Exp. Med. 202, 1051-1061 (2005).

19. Al-Lamki, R.S. et al. TL1A both promotes and protects from renal inflammation and injury. J. Am. Soc. Nephrol. 19, 953-960 (2008).

20. Wang, E.C., Thern, A., Denzel, A., Kitson, J., Farrow, S.N. \& Owen, M.J. DR3 regulates negative selection during thymocyte development. Mol. Cell Biol. 21, 3451-3461 (2001).

21. Izcue, A., Coombes, J.L. \& Powrie, F. Regulatory lymphocytes and intestinal inflammation. Annu. Rev. Immunol. 27, 313-338 (2009).

22. Fuss, I.J. et al. Nonclassical CD1d-restricted NKT cells that produce IL-13 characterize an atypical Th2 response in ulcerative colitis. J. Clin. Invest. 113, 1490-1497 (2004).

23. Rakoff-Nahoum, S., Hao, L. \& Medzhitov, R. Role of toll-like receptors in spontaneous commensal-dependent colitis. Immunity 25, 319-329 (2006).

24. Powrie, F. Immune regulation in the intestine: a balancing act between effector and regulatory T cell responses. Ann. NY Acad. Sci. 1029, 132-141 (2004).

25. Zhou, D. et al. Lysosomal glycosphingolipid recognition by NKT cells. Science 306, 1786-1789 (2004).

26. Terabe, M. \& Berzofsky, J.A. NKT cells in immunoregulation of tumor immunity: a new immunoregulatory axis. Trends Immunol. 28, 491-496 (2007).

27. Zeissig, S., Kaser, A., Dougan, S.K., Nieuwenhuis, E.E. \& Blumberg, R.S. Role of NKT cells in the digestive system. III. Role of NKT cells in intestinal immunity. Am. J. Physiol. Gastrointest. Liver Physiol. 293, G1101-G1105 (2007).

28. van der Vliet, H.J. et al. Circulating V(alpha24+) Vbeta11+ NKT cell numbers are decreased in a wide variety of diseases that are characterized by autoreactive tissue damage. Clin. Immunol. 100, 144-148 (2001).

29. Grose, R.H., Thompson, F.M., Baxter, A.G., Pellicci, D.G. \& Cummins, A.G. Deficiency of invariant NKT cells in Crohn's disease and ulcerative colitis. Dig. Dis. Sci. 52, 1415-1422 (2007).

30. Korn, T. et al. Myelin-specific regulatory T cells accumulate in the CNS but fail to control autoimmune inflammation. Nat. Med. 13, 423-431 (2007).

31. Sun, C.M. et al. Small intestine lamina propria dendritic cells promote de novo generation of Foxp3 T reg cells via retinoic acid. J. Exp. Med. 204, 1775-1785 (2007).

32. Coombes, J.L. et al. A functionally specialized population of mucosal CD103+ DCs induces Foxp3+ regulatory T cells via a TGF-beta and retinoic acid-dependent mechanism. J. Exp. Med. 204, 1757-1764 (2007).

33. Mills, K.H. Regulatory T cells: friend or foe in immunity to infection? Nat. Rev. Immunol. 4, 841-855 (2004).

34. Takedatsu, H. et al. TL1A (TNFSF15) regulates the development of chronic colitis by modulating both T-helper 1 and T-helper 17 activation. Gastroenterology 135, 552-567 (2008).

35. Dieleman, L.A., Ridwan, B.U., Tennyson, G.S., Beagley, K.W., Bucy, R.P. \& Elson, C.O. Dextran sulfate sodium-induced colitis occurs in severe 
combined immunodeficient mice. Gastroenterology 107, 1643-1652 (1994).

36. Hale, L.P. \& Cianciolo, G. Treatment of experimental colitis in mice with LMP-420, an inhibitor of TNF transcription. J. Inflamm. (Lond). 5, 4 (2008).

37. Kontoyiannis, D., Pasparakis, M., Pizarro, T., Cominelli, F. \& Kollias, G. Impaired on/off regulation of TNF biosynthesis in mice lacking TNF AU-rich elements: implications for joint and gut-associated immunopathologies. Immunity 10, 387-398 (1999).

38. Rivera-Nieves, J. et al. Emergence of perianal fistulizing disease in the SAMP1/YitFc mouse, a spontaneous model of chronic ileitis. Gastroenterology 124, 972-982 (2003).

39. Shaikh, R.B. et al. Constitutive expression of LIGHT on T cells leads to lymphocyte activation, inflammation, and tissue destruction. J. Immunol. 167, 6330-6337 (2001).

40. Corry, D.B. IL-13 in allergy: home at last. Curr. Opin. Immunol. 11, 610-614 (1999).

41. McKenzie, G.J., Bancroft, A., Grencis, R.K. \& McKenzie, A.N. A distinct role for interleukin-13 in Th2-cell-mediated immune responses. Curr. Biol. 8, 339-342 (1998).

42. Zhao, A. et al. Dependence of IL-4, IL-13, and nematode-induced alterations in murine small intestinal smooth muscle contractility on Stat6 and enteric nerves. J. Immunol. 171, 948-954 (2003).

43. Morimoto, M. et al. Functional importance of regional differences in localized gene expression of receptors for IL-13 in murine gut. J. Immunol. 176, 491-495 (2006).

44. Jarry, A. et al. Mucosal IL-10 and TGF-beta play crucial roles in preventing LPS-driven, IFN-gamma-mediated epithelial damage in human colon explants. J. Clin. Invest. 118, 1132-1142 (2008).

45. Urban, J.F. Jr et al. IL-13, IL-4Ralpha, and Stat6 are required for the expulsion of the gastrointestinal nematode parasite Nippostrongylus brasiliensis. Immunity 8, 255-264 (1998).

46. Finkelman, F.D., Wynn, T.A., Donaldson, D.D. \& Urban, J.F. The role of IL-13 in helminth-induced inflammation and protective immunity against nematode infections. Curr. Opin. Immunol. 11, 420-426 (1999).

47. Li, H., Sim, T.C. \& Alam, R. IL-13 released by and localized in human basophils. J. Immunol. 156, 4833-4838 (1996).
48. Mcdermott, J., Humphreys, N., Forman, S., Donaldson, D. \& Grencis, R. Intraepithelial NK cell-derived IL-13 induces intestinal pathology associated with nematode infection. J. Immunol. 175, 3207 (2005).

49. Moro, K. et al. Innate production of $T(H) 2$ cytokines by adipose tissueassociated c-Kit(+)Sca-1(+) lymphoid cells. Nature 463, 540-544 (2010).

50. Neill, D.R. et al. Nuocytes represent a new innate effector leukocyte that mediates type-2 immunity. Nature 464, 1367-1370 (2010).

51. Thornton, A.M., Donovan, E.E., Piccirillo, C.A. \& Shevach, E.M. Cutting edge: IL-2 is critically required for the in vitro activation of CD4+CD25+ T cell suppressor function. J. Immunol. 172, 6519-6523 (2004).

52. Coombes, J.L. \& Maloy, K.J. Control of intestinal homeostasis by regulatory T cells and dendritic cells. Semin. Immunol. 19, 116-126 (2007).

53. Becker, C. et al. Cutting edge: IL-23 cross-regulates IL-12 production in T cell-dependent experimental colitis. J. Immunol. 177, 2760-2764 (2006).

54. Fichtner-Feigl, S., Strober, W., Geissler, E.K. \& Schlitt, H.J. Cytokines mediating the induction of chronic colitis and colitis-associated fibrosis. Mucosal Immunol. 1 (Suppl 1), S24-S27 (2008).

55. Neurath, M.F., Fuss, I., Kelsall, B.L., Stuber, E. \& Strober, W. Antibodies to interleukin 12 abrogate established experimental colitis in mice. J. Exp. Med. 182, 1281-1290 (1995).

56. Zhang, Z., Zheng, M., Bindas, J. \& Schwarzenberger, P. Critical role of IL-17 receptor signaling in acute TNBS-induced colitis. Inflamm. Bowel Dis. 12, 382-388 (2006).

57. Fuss, I.J. et al. Both IL-12p70 and IL-23 are synthesized during active Crohn's disease and are down-regulated by treatment with anti-IL-12 p40 monoclonal antibody. Inflamm. Bowel Dis. 12, 9-15 (2006).

58. Heller, F. et al. Interleukin-13 is the key effector Th2 cytokine in ulcerative colitis that affects epithelial tight junctions, apoptosis, and cell restitution. Gastroenterology 129, 550-564 (2005).

59. Brocker, T., Riedinger, M. \& Karjalainen, K. Targeted expression of major histocompatibility complex (MHC) class II molecules demonstrates that dendritic cells can induce negative but not positive selection of thymocytes in vivo. J. Exp. Med. 185, 541-550 (1997).

60. Lefrancois, L. \& Lycke, N. Isolation of mouse small intestinal intraepithelial lymphocytes, Peyer's patch, and lamina propria cells. Curr. Protoc. Immunol. Chapter 3: Unit 3.19.1-3.19.16 (2001). 